\title{
A Quantum Weak Energy Inequality for Dirac Fields in Curved Spacetime
}

\author{
Christopher J. Fewster ${ }^{1}$ and Rainer Verch ${ }^{2}$ \\ ${ }^{1}$ Department of Mathematics, University of York, \\ Heslington, York YO10 5DD, United Kingdom. \\ e-mail: cjf3@york.ac.uk \\ ${ }^{2}$ Institut für Theoretische Physik,Universität Göttingen, \\ Bunsenstr. 9, D-37073 Göttingen, Germany. \\ e-mail: verch@theorie.physik.uni-goettingen.de
}

May 21, 2001

\begin{abstract}
Quantum fields are well known to violate the weak energy condition of general relativity: the renormalised energy density at any given point is unbounded from below as a function of the quantum state. By contrast, for the scalar and electromagnetic fields it has been shown that weighted averages of the energy density along timelike curves satisfy 'quantum weak energy inequalities' (QWEIs) which constitute lower bounds on these quantities. Previously, Dirac QWEIs have been obtained only for massless fields in two-dimensional spacetimes. In this paper we establish QWEIs for the Dirac and Majorana fields of mass $m \geq 0$ on general four-dimensional globally hyperbolic spacetimes, averaging along arbitrary smooth timelike curves with respect to any of a large class of smooth compactly supported positive weights. Our proof makes essential use of the microlocal characterisation of the class of Hadamard states, for which the energy density may be defined by point-splitting.
\end{abstract}




\section{Introduction}

In general relativity, it is customary to assume that the stress-energy tensor satisfies one or more of the classical energy conditions; the weak energy condition, for example, being the assertion that the energy density measured by any observer is nonnegative. The primary motivation behind these energy conditions is that they ensure that gravity acts as an attractive force (in the sense of focussing geodesic congruences) in accordance with our experience of gravitation on a wide range of scales. It is therefore natural to assume that physically reasonable forms of classical matter obey such conditions, and to regard matter theories (such as the nonminimally coupled scalar field [4, 43, 12, 17]) violating such conditions as being of questionable physical significance on many scales. Moreover, the energy conditions have proved to be of great value in obtaining deep results in classical general relativity, such as the positive mass and singularity theorems [39, 48, 21].

However, it is well known that all the pointwise energy conditions are violated in quantum field theory. Indeed, Epstein, Glaser and Jaffe [6] proved that no Wightman field theory on Minkowski space can admit a (nontrivial) energy density observable whose expectation values are bounded from below and vanish in the Minkowski vacuum state. Moreover, in linear field theories (both in flat and curved spacetimes) it is easy to construct states whose energy density at a given point may be tuned to be arbitrarily negative [3, 26]. This raises the possibility that quantum matter might be used to construct spacetimes with exotic properties, such as traversable wormholes [18] or so-called 'warp drive' spacetimes [33], usually excluded by the classical energy conditions. One might also ask whether the conclusions of the singularity theorems remain valid for quantum matter. Furthermore, it is necessary to understand how classical matter contrives to obey the classical energy conditions, given that its fundamental constituents need not.

One profitable line of enquiry, starting with the work of Ford [14], has been to investigate weighted averages of the renormalised energy density along the worldline of an observer, or over a small spacetime region. It turns out that the expectation values of these averaged observables are bounded from below independently of the state, and such bounds have been developed in successively greater generality over the last few years [16, 34, 11, 7, 9, 22, 8]. Most recently, one of us [8] has established the existence of such bounds (and given an explicit, though not optimal, lower bound) for the minimally coupled real linear scalar field in any globally hyperbolic spacetime, in the case where averaging is performed with respect to proper time along any smooth timelike curve using an arbitrary smooth compactly supported positive weight belonging to the class:

$$
\mathscr{W}=\left\{f \in C_{0}^{\infty}(\mathbb{R}) \mid f(\tau)=g(\tau)^{2} \text { for some real-valued } g \in C_{0}^{\infty}(\mathbb{R})\right\} .
$$

The constraints imposed by these lower bounds have been called 'quantum inequalities' by various authors. However, as we hope to discuss elsewhere, there seem to be strong parallels between the phenomena discussed above and various situations arising in quantum mechanics. Indeed, quantum inequalities appear to be a widespread feature of quantum theory as a whole, stemming ultimately from the uncertainty principle. In this light, we adopt the more specific terminology 'quantum weak energy inequality' (or QWEI) in relation to lower bounds on the renormalised energy density of a quantum field.

\footnotetext{
${ }^{1}$ See the remarks following Theorem 4.1 for a brief discussion of this class.
} 
There are also related constraints which demand that the integral of the energy density over any complete (i.e. inextendible) smooth timelike or lightlike ('null'-) curve be nonnegative. These are called the 'averaged weak energy condition' (AWEC) or 'averaged null energy condition' (ANEC), respectively; they may be viewed as a limiting case of QWEIs when the weight function ( $f$ in eqn. (1.2) below) against which the energy density is integrated along a timelike or lightlike curve approaches the unit function. Historically, it was first pointed out in a work by Tipler [41 that suitable versions of AWEC or ANEC imply singularity theorems in general relativity similar to those which one obtains from pointwise positivity conditions on the energy density. Subsequently, the question of whether quantum fields obey AWEC or ANEC has been investigated in a number of works [26, 13, 46, 49, 50, 15, 12, 42. Most of these references treat linear quantum fields; 42 establishes ANEC for general (axiomatic) quantum field theory in two-dimensional Minkowski spacetime. A study of the interrelations between averaged energy conditions and QWEIs is contained in [15]. We refer to [12] for further discussion and review of averaged energy conditions.

QWEIs place stringent constraints on attempts to generate exotic spacetimes [18, 33] and may open a route towards proving results analogous to the singularity theorems for quantum matter [49, 50]. To date, however, most QWEI results have been obtained for scalar field theories, while the more physically interesting electromagnetic and Dirac fields have received comparatively little attention. Ford and Roman have considered the electromagnetic field in Minkowksi space [16] and shown that a QWEI holds for averaging against the Lorentzian weight $f(\tau)=\tau_{0} /\left[\pi\left(\tau^{2}+\tau_{0}^{2}\right)\right]$ along timelike geodesics; this result was generalised to static trajectories in static spacetimes by Pfenning [31], who has also removed the restriction to Lorentzian weights [32]. It is reasonable to suppose that even more general QWEIs may be obtained for this case.

Both the scalar and electromagnetic fields have the property that the classical energy density is manifestly nonnegative, a fact which underpins all the results on these fields. The Dirac field is technically very different in that the 'classical' energy density is unbounded both from above and below; in second quantization, renormalisation serves the dual purpose of restoring finiteness and imposing positivity of the Hamiltonian. This problem appears to have restricted progress on the Dirac field to date. The main contribution has been that of Vollick, who established a QWEI for Dirac fields in two-dimensional spacetimes 45 by converting the problem to one involving a scalar field and then adapting arguments due to Flanagan [11]. There seems little prospect of generalising this argument beyond the two-dimensional setting. In four dimensions, Vollick has also given explicit examples of states with locally negative energy densities 44 and demonstrated that the resulting energy densities nonetheless obey QWEIs modelled on those for the scalar field.

In this paper we establish a general QWEI for massive or massless Dirac fields on four-dimensional| $\left.\right|^{2}$ globally hyperbolic spacetimes. To be more specific, let $\gamma$ be a smooth timelike curve, parametrized by its proper time $\tau$, in a globally hyperbolic spacetime $(M, \boldsymbol{g})$. Let $\omega_{0}$ be a given (but arbitrary) Hadamard state of the Dirac field on $(M, \boldsymbol{g})$. The state $\omega_{0}$ is used as a 'reference state' to define the expected normal ordered energy

\footnotetext{
${ }^{2}$ The restriction to four dimensions is purely for convenience: our methods would apply in more general dimensions.

${ }^{3}$ See Sec. 2 for a brief review of the concepts used here.
} 
density $\left\langle: T_{00}:\right\rangle_{\omega}$ for any other Hadamard state $\omega$. Our main result, Theorem 4.1 asserts that

$$
\inf _{\omega} \int d \tau\left\langle: T_{00}:\right\rangle_{\omega}(\gamma(\tau)) f(\tau)>-\infty
$$

where the infimum is taken over the class of Hadamard states and $f$ belongs to the class $\mathscr{W}$. In principle our arguments yield an explicit lower bound for the left-hand side of (1.2). This expression is unfortunately not particularly enlightening and is not expected to be sharp. Let us note that (1.2) remains true if the normal ordered energy density is replaced by the renormalised energy density, as these two quantities differ by a smooth function.

The plan of the paper is as follows. We begin, for completeness and to fix notation, by reviewing the theory of the quantized Dirac field in Sec. 2. Particular attention is given to the class of Hadamard states, which may be characterised by a microlocal spectrum condition on the wave-front set of the two-point function. This formulation of the Hadamard condition is technically convenient and allows us to bring the tools of microlocal analysis to bear. Sec. 3 explains how the normal ordered energy density may be constructed by point-splitting.

The proof of our QWEI begins in Sec. 4, using the following strategy. The averaged normal ordered energy density is first expressed as an integral over $\mathbb{R}^{2}$; decomposing this integral according to the quadrants of $\mathbb{R}^{2}$, each piece is then split further into four using a decomposition induced by the reference state $\omega_{0}$. All but two of the resulting sixteen contributions can be bounded (both above and below) using estimates obtained in Sec. 5 . The remaining terms are then expressed in the form $\mathcal{R}=\lim _{\Lambda \rightarrow+\infty} \operatorname{Tr} J_{\Lambda} W$ where $J_{\Lambda}$ and $W$ are self-adjoint and $J_{\Lambda}$ is independent of $\omega$. The parameter $\Lambda \in \mathbb{R}^{+}$defines a cut-off, used to avoid domain problems. We prove that $W$ is positive and trace-class with bounded trace as $\omega$ varies. To conclude that $\mathcal{R}$ is bounded below, it then suffices to establish that the operators $J_{\Lambda}$ are bounded below uniformly in $\Lambda$. This is accomplished in Sec. 6, completing the proof of our QWEI. In Sec. 7 we briefly describe how our arguments can also be applied to the Majorana field.

Conventions: The metric signature is $(+,-,-,-)$. Lower (resp. upper) case Latin characters from the beginning of the alphabet will label tetrad (resp. spinor) indices. Tetrad indices run from 0 to 3 , and we will use $j, k$ to label the spatial components $1,2,3$. The summation convention will be used throughout the paper except where otherwise indicated. Units with $c=\hbar=1$ are adopted.

The Fourier transform of an integrable function $f$ on $\mathbb{R}^{n}$ will be defined using the nonstandard convention $\widehat{f}(k)=\int d^{n} x \mathrm{e}^{i k x} f(x)$, with inverse $\check{h}(x)=(2 \pi)^{-n} \int d^{n} k \mathrm{e}^{-i k x} h(k)$. The Fourier transform of a distribution $u$ with compact support is $\widehat{u}(k)=u\left(e_{k}\right)$ with $e_{k}(x)=\mathrm{e}^{i k x}$.

Given a Lorentzian manifold $(M, \boldsymbol{g}), \mathscr{D}^{\prime}(M)$ will denote the space of distributions on $M$ as defined in $\S 6.3$ of [24]. Thus if $u \in \mathscr{D}^{\prime}(M)$ there is, for each chart $(U, \kappa)$ in $M$, a distribution $u_{\kappa} \in \mathscr{D}^{\prime}(\kappa(U))$ such that

$$
u(f)=u_{\kappa}\left((\sqrt{-|\boldsymbol{g}|} f) \circ \kappa^{-1}\right) \quad \forall f \in C_{0}^{\infty}(U)
$$

\footnotetext{
${ }^{4}$ The factor of $\sqrt{-|\boldsymbol{g}|}$ is used to identify test functions with test densities, on which $u$ strictly speaking acts.
} 
and so that $u_{\kappa}=\left(\kappa^{\prime} \circ \kappa^{-1}\right)^{*} u_{\kappa}^{\prime}$ in $\kappa\left(U \cap U^{\prime}\right)$ for any other chart $\left(U^{\prime}, \kappa^{\prime}\right)$. We will also (and usually) write $u \circ \kappa^{-1}$ for $u_{\kappa}$. Spinor and cospinor distributions will be defined in an analogous fashion, with the convention that a spinor distribution acts on test cospinor fields, and vice versa.

\section{The Quantized Dirac Field}

\subsection{Geometrical preliminaries}

In order to make the present paper sufficiently self-contained, we need to summarize a few basic facts about the geometry of spinor fields in curved spacetimes. We will follow Dimock's work [5] to large extent.

We will consider Dirac fields in a four-dimensional globally hyperbolic spacetime $(M, \boldsymbol{g})$. To begin with, we recall that a globally hyperbolic spacetime is a Lorentzian spacetime admitting a Cauchy surface, the latter being a smooth hypersurface in $M$ which is intersected exactly once by each inextendible causal curve in $(M, \boldsymbol{g})$. We will also suppose that $(M, \boldsymbol{g})$ is orientable and time-orientable, and that such orientations have been chosen. Then $(M, \boldsymbol{g})$ possesses a spin-structure, that is, there is a principal fibre bundle $S(M, \boldsymbol{g})$ having $\mathrm{SL}(2, \mathbb{C})$ as structure group, acting from the right, together with a 2-1 fibre-bundle homomorphism $\psi: S(M, \boldsymbol{g}) \rightarrow F(M, \boldsymbol{g})$ which projects $S(M, \boldsymbol{g})$ onto the frame bundle $F(M, \boldsymbol{g})$. That is to say, $\psi$ preserves base-points and obeys

$$
\psi \circ R_{\mathrm{s}}=R_{\Lambda(\mathbf{s})} \circ \psi
$$

where $R$ denotes the right action of the structure groups on the principal fibre bundles involved and $\operatorname{SL}(2, \mathbb{C}) \ni \mathbf{s} \mapsto \Lambda(\mathbf{s}) \in \mathscr{L}_{+}^{\uparrow}$ is the covering projection onto the proper orthochronous Lorentz group. We recall that $F(M, \boldsymbol{g})$ is the bundle of oriented and timeoriented tetrads $\left(e_{0}, e_{1}, e_{2}, e_{3}\right)$, so that $\boldsymbol{g}\left(e_{a}, e_{b}\right)=\eta_{a b}$ with $\eta_{a b}=\operatorname{diag}(1,-1,-1,-1)$ where $e_{0}$ is timelike and future-pointing and the tetrad is given the orientation of $M$.

Moreover, a collection of $4 \times 4$-matrices $\gamma_{0}, \ldots, \gamma_{3}$ is called a set of Dirac matrices if

$$
\gamma_{a} \gamma_{b}+\gamma_{b} \gamma_{a}=2 \eta_{a b} \cdot \mathbb{1}
$$

A theorem due to Pauli states that, if $\gamma_{0}, \ldots, \gamma_{3}$ and $\gamma_{0}^{\prime}, \ldots, \gamma_{3}^{\prime}$ are two sets of Dirac matrices, then there is an invertible matrix $M$ so that $\gamma_{a}=M \gamma_{a}^{\prime} M^{-1}$. Any set of Dirac matrices $\gamma_{0}, \ldots, \gamma_{3}$ is connected to the covering $\operatorname{SL}(2, \mathbb{C}) \stackrel{\Lambda(\cdot)}{\longrightarrow} \mathscr{L}_{+}^{\uparrow}$ in the following way. Let $\operatorname{Spin}(1,3)$ consist of all unimodular $4 \times 4$-matrices $S$ so that

$$
S \gamma_{a} S^{-1}=\gamma_{b} \Lambda_{a}^{b}
$$

holds for some real numbers $\Lambda^{b}{ }_{a}=\Lambda^{b}{ }_{a}(S)$. It follows from the defining properties of Dirac matrices that $\Lambda^{b}{ }_{a}(S)$ is contained in the Lorentz group. The restriction of the map $S \mapsto \Lambda^{b}{ }_{a}(S)$ in (2.3) to $\operatorname{Spin}_{0}(1,3)$, the unit connected component of $\operatorname{Spin}(1,3)$, is a group homomorphism with range $\mathscr{L}_{+}^{\uparrow}$, and thus $\operatorname{Spin}_{0}(1,3)$ is isomorphic to $\operatorname{SL}(2, \mathbb{C})$.

Sometimes it is useful to distinguish sets of Dirac matrices with certain properties. One says that a set $\gamma_{0}, \ldots, \gamma_{3}$ of Dirac matrices belongs to a standard representation if

$$
\gamma_{0}^{*}=\gamma_{0} \quad \text { and } \quad \gamma_{k}^{*}=-\gamma_{k} .
$$


Here, $\gamma_{a}^{*}$ is the hermitean adjoint of $\gamma_{a}$. A set of Dirac matrices which belongs to a standard representation and has the additional property that the complex conjugate matrices fulfill

$$
\bar{\gamma}_{a}=-\gamma_{a}
$$

is said to belong to a Majorana representation.

We will now suppose that we are given a globally hyperbolic spacetime $(M, \boldsymbol{g})$ together with a spin-structure $(S(M, \boldsymbol{g}), \psi)$ and a set of Dirac matrices $\gamma_{0}, \ldots, \gamma_{3}$ which we will assume, for the sake of notational simplicity, to belong to a standard representation. (We note, however, that everything which follows could also be carried out in a similar way without that assumption.) As was pointed out above, via the isomorphism $\operatorname{Spin}_{0}(1,3) \simeq$ $\mathrm{SL}(2, \mathbb{C}), \mathbb{C}^{4}$ carries a representation of the universal covering group of $\mathscr{L}_{+}^{\uparrow}$ which is given by the action of the matrices $S$ in $\operatorname{Spin}_{0}(1,3)$ on vectors in $\mathbb{C}^{4}$. Via $\operatorname{Spin}_{0}(1,3) \simeq \mathrm{SL}(2, \mathbb{C})$, we can also regard $S(M, \boldsymbol{g})$ as a $\operatorname{Spin}_{0}(1,3)$-principal bundle and form the associated vector bundle

$$
D M=S(M, \boldsymbol{g}) \ltimes_{\operatorname{Spin}_{0}(1,3)} \mathbb{C}^{4} .
$$

That is, the fibre of $D M$ at $p \in M$ consists of the orbits

$$
\left[\mathbf{s}_{p}, x\right]=\left\{\left(R_{S}^{-1} \mathbf{s}_{p}, S x\right): S \in \operatorname{Spin}_{0}(1,3)\right\}
$$

for $\mathbf{s}_{p} \in S(M, \boldsymbol{g})_{p}$ and $x \in \mathbb{C}^{4}$. There is a fibrewise left action of $\operatorname{Spin}_{0}(1,3)$ on $D M$ by $L_{S}\left[\mathbf{s}_{p}, x\right]=\left[\mathbf{s}_{p}, S x\right]$. Elements in $D M$ are called spinors, and elements in the dual bundle $D^{*} M$ are called cospinors. Moreover, if $E$ is a (local) section in $S(M, \boldsymbol{g})$, then it induces on one hand a tetrad field $\left(e_{0}, \ldots, e_{3}\right)=\psi \circ E$, i.e. a (local) smooth section in $F(M, \boldsymbol{g})$, via the spin-structure, and on the other hand it induces a set $\left(E_{A}\right)_{A=1}^{4}$ of (local) smooth sections in $D M$, defined by

$$
E_{A}=\left[E, b_{A}\right]
$$

where $b_{1}, \ldots, b_{4}$ is the standard basis in $\mathbb{C}^{4}$. There are corresponding dual tetrad fields $\left(e^{0}, \ldots, e^{3}\right)$ defined by $e^{b}\left(e_{a}\right)=\delta_{a}^{b}$ and $\left(E^{B}\right)_{B=1}^{4}$ defined by $E^{B}\left(E_{A}\right)=\delta_{A}^{B}$. The $e^{b}$ are smooth sections in $T^{*} M$, and the $E^{B}$ are smoth sections in $D^{*} M$, the dual bundle to $D M$. We shall denote by $C^{\infty}(D M)$ and $C^{\infty}\left(D^{*} M\right)$ the sets of smooth sections in $D M$ and $D^{*} M$, respectively. The notation for smooth sections in $T M$ and $T^{*} M$ will be similar.

With respect to the given set of Dirac matrices, one can define a section $\gamma$ in $C^{\infty}\left(T^{*} M\right) \otimes$ $C^{\infty}(D M) \otimes C^{\infty}\left(D^{*} M\right)$, i.e. a mixed spinor-tensor field, by setting its components $\gamma_{b}{ }_{B}{ }_{B}$ in the induced frame $e^{b} \otimes E_{A} \otimes E^{B}$ to be equal to the matrix elements $\left(\gamma_{b}\right)^{A}{ }_{B}$ of $\gamma_{b}$. This definition is independent of the induced frames, i.e. independent of the chosen (local) section $E$ in $S(M, \boldsymbol{g})$. (Once the set of Dirac matrices $\gamma_{0}, \ldots, \gamma_{3}$ is given, $\gamma$ encodes the spin-structure at the level of $D M$.)

Moreover, there is an anti-linear isomorphism $D M \rightarrow D^{*} M$ induced by forming the Dirac adjoint: If $u=u^{A} E_{A}$ is a spinor, one can assign to it a cospinor $u^{+}=u_{B}^{+} E^{B}$ with components

$$
u_{B}^{+}=\overline{u^{A}} \gamma_{0 A B}
$$


where $\gamma_{0 A B}$ are the matrix elements of $\gamma_{0}$. This assignment possesses an inverse (denoted by the same symbol), where a cospinor $v=v_{B} E^{B}$ is mapped to a spinor $v^{+}$having components $v^{+A}=\gamma_{0}{ }^{A B} \overline{v_{B}}$. Again, $\gamma_{0}{ }^{A B}$ are the matrix elements of $\gamma_{0}$. The operation of taking the Dirac adjoint gives rise to anti-linear isomorphisms between $C^{\infty}(D M)$ and $C^{\infty}\left(D^{*} M\right)$ in the obvious manner.

The metric-induced covariant derivative $\nabla$ on $C^{\infty}(T M)$ induces a covariant derivative, also denoted by $\nabla$, on $C^{\infty}(D M)$. If $\left(e_{0}, \ldots, e_{3}\right)$ and $\left(E_{A}\right)_{A=1}^{3}$ are induced by a section $E$ in $S(M, \boldsymbol{g})$ and $f=f^{A} E_{A}$ is a local section in $D M$, then

$$
\nabla f=\nabla_{b} f^{A}\left(e^{b} \otimes E_{A}\right) \in C^{\infty}\left(T^{*} M\right) \otimes C^{\infty}(D M)
$$

has the frame components

$$
\nabla_{b} f^{A}=\partial_{b} f^{A}+\sigma_{b}{ }^{A}{ }_{B} f^{B}, \quad \text { where } \quad \partial_{b} f^{A}=d f^{A}\left(e_{b}\right), \quad \sigma_{b}{ }^{A}{ }_{B}=-\frac{1}{4} \Gamma_{b d}^{a} \gamma_{a}{ }^{A}{ }_{C} \gamma^{d C}{ }_{B}
$$

Here, $d f^{A}$ is the differential of the function $f^{A}$, and we read the components of the Dirac matrices on the right hand side while $\Gamma_{b d}^{a}$ are Christoffel's connection coefficients, defined by

$$
\nabla k=\left(\partial_{b} k^{a}+\Gamma_{b d}^{a} k^{d}\right) e^{b} \otimes e_{a}
$$

for $k=k^{b} e_{b} \in C^{\infty}(T M)$.

The covariant derivative $\nabla$ can be extended to cospinor fields and mixed spinor-tensor fields by requiring the Leibniz rule and commutativity with contractions. Thus, if $h=$ $h_{B} E^{B}$ is a cospinor field, then $\nabla h=\nabla_{b} h_{B} e^{b} \otimes E^{B}$ has the components

$$
\nabla_{b} h_{B}=\partial_{b} h_{B}-h_{C} \sigma_{b}^{C}{ }_{B}
$$

It follows that $\nabla \gamma=0$.

\subsection{The Dirac Equation}

The Dirac-operator $\nabla$ is a first order differential operator taking spinor fields to spinor fields, or cospinor fields to cospinor fields; it is defined as the action of the covariant derivative followed by contraction with the spinor-tensor $\gamma$. More precisely, if $f=f^{A} E_{A} \in$ $C^{\infty}(D M)$ and $h=h_{B} E^{B} \in C^{\infty}\left(D^{*} M\right)$, then

$$
\begin{aligned}
& \not \nabla f=(\not \nabla f)^{A} E_{A}=\eta^{a b} \gamma_{a}{ }^{A}{ }_{B} \nabla_{b} f^{B} E_{A}, \\
& \nabla h=(\not \nabla h)_{B} E^{B}=\eta^{a b} \nabla_{b} h_{C} \gamma_{a}{ }^{C}{ }_{B} E^{B} .
\end{aligned}
$$

An important property of the Dirac operator is that it commutes with taking the Dirac adjoint, i.e.

$$
(\not \nabla f)^{+}=\not \nabla f^{+} \quad \text { and } \quad(\not \nabla h)^{+}=\not \nabla h^{+}
$$

for $f \in C^{\infty}(D M)$ and $h \in C^{\infty}\left(D^{*} M\right)$. 
The Dirac equation is the following first order partial differential equation for spinor fields $u \in C^{\infty}(D M)$ or for cospinor fields $v \in C^{\infty}\left(D^{*} M\right)$ :

$$
\begin{aligned}
(-i \not \nabla+m) u & =0, \\
(i \not \nabla+m) v & =0,
\end{aligned}
$$

where $m \geq 0$ is a constant. Then

$$
P=(i \not \nabla+m)(-i \not \nabla+m)
$$

is the Lichnerowicz wave operator on spinors or cospinors. It is a second order wave operator which has metric principal part, and owing to global hyperbolicity of $(M, \boldsymbol{g})$ this implies that the Cauchy problem for the corresponding wave equations is well-posed and that $P$ possesses uniquely determined advanced and retarded fundamental solutions. As shown in [5], this implies that the Dirac operators $-i \nabla \nabla+m$ on spinor fields and $i \nabla \nabla+m$ on cospinor fields possess uniquely determined pairs of advanced $(-)$ and retarded $(+)$ fundamental solutions $S_{\mathrm{sp}}^{ \pm}$and $S_{\text {cosp }}^{ \pm}$, respectively: This means that, for the spinor case,

$$
S_{\mathrm{sp}}^{ \pm}: C_{0}^{\infty}(D M) \rightarrow C^{\infty}(D M)
$$

are continuous linear maps so that

$$
(-i \not \nabla+m) S_{\mathrm{sp}}^{ \pm} u=u=S_{\mathrm{sp}}^{ \pm}(-i \not \nabla+m) u
$$

holds for all $u \in C_{0}^{\infty}(D M)$ and, moreover, $\operatorname{supp} S_{\mathrm{sp}}^{ \pm} u$ is contained in the causal future $(+) /$ causal past $(-)$ of $\operatorname{supp} u$. (We note that our convention concerning advanced/retarded fundamental solutions is opposite to that in [38.) The cospinor case is analogous. Then one defines the retarded-minus-advanced fundamental solutions

$$
S_{\mathrm{sp}}=S_{\mathrm{sp}}^{+}-S_{\mathrm{sp}}^{-} \quad \text { and } \quad S_{\mathrm{cosp}}=S_{\mathrm{cosp}}^{+}-S_{\mathrm{cosp}}^{-}
$$

In order to quantize the Dirac field, it is very convenient to 'double' the system by taking pairs of spinor fields and cospinor fields together, as was done in the references [28, 29, 23. We give here an equivalent version which makes contact with the notation used in [38]. To this end, let us denote $C_{0}^{\infty}(D M)$ by $\mathscr{D}_{\text {sp }}$ and $C_{0}^{\infty}\left(D^{*} M\right)$ by $\mathscr{D}_{\text {cosp }}$, and define the doubled space $\mathscr{D}_{\text {double }}=\mathscr{D}_{\text {cosp }} \oplus \mathscr{D}_{\mathrm{sp}}$. On $\mathscr{D}_{\text {double }}$ we introduce the sesquilinear form

$$
\left(\left[\begin{array}{c}
h_{1} \\
f_{1}
\end{array}\right],\left[\begin{array}{c}
h_{2} \\
f_{2}
\end{array}\right]\right)=\left\langle f_{1}^{+}, f_{2}\right\rangle-\left\langle h_{1}, h_{2}^{+}\right\rangle
$$

for $h_{1}, h_{2} \in \mathscr{D}_{\operatorname{cosp}}$ and $f_{1}, f_{2} \in \mathscr{D}_{\mathrm{sp}}$, where for $v \in \mathscr{D}_{\text {cosp }}$ and $u \in \mathscr{D}_{\mathrm{sp}}$ we employ the dual pairing

$$
\langle v, u\rangle=\int_{M} d \mu_{\boldsymbol{g}}(p) v_{p}\left(u_{p}\right)
$$

with $d \mu_{\boldsymbol{g}}$ denoting the canonical 4-volume form induced by the metric $\boldsymbol{g}$ on $M$. This dual pairing also embeds $\mathscr{D}_{\text {cosp }}$ in the (topological) dual space $\mathscr{D}_{\mathrm{sp}}^{\prime}$ of $\mathscr{D}_{\mathrm{sp}}$, and vice versa, 
embeds $\mathscr{D}_{\text {sp }}$ in $\mathscr{D}_{\text {cosp }}^{\prime}$. The sesquilinear form $(.,$.$) is non-degenerate, but not positive. A$ useful relation is

$$
\left\langle S_{\mathrm{cosp}} h, f\right\rangle=-\left\langle h, S_{\mathrm{sp}} f\right\rangle
$$

for $h \in \mathscr{D}_{\text {cosp }}$ and $f \in \mathscr{D}_{\text {sp }}$.

Let us define the conjugate-linear isomorphism $\Gamma: \mathscr{D}_{\text {double }} \rightarrow \mathscr{D}_{\text {double }}$, playing the role of a charge-conjugation, by

$$
\Gamma\left(\left[\begin{array}{l}
h \\
f
\end{array}\right]\right)=\left[\begin{array}{l}
f^{+} \\
h^{+}
\end{array}\right]
$$

Then one finds that $\Gamma$ is a skew-conjugation with respect to $(.,$.$) , namely it holds that$

$$
\left(\Gamma F_{1}, \Gamma F_{2}\right)=-\left(F_{2}, F_{1}\right) \quad \forall F_{1}, F_{2} \in \mathscr{D}_{\text {double }} .
$$

Now we introduce the following 'doubled' operators on $\mathscr{D}_{\text {double }}$ :

$$
\begin{gathered}
D_{\triangleright}=\left(\begin{array}{cc}
-\nabla+i m & 0 \\
0 & \nabla+i m
\end{array}\right), \quad D_{\triangleleft}=\left(\begin{array}{cc}
-\nabla-i m & 0 \\
0 & \nabla-i m
\end{array}\right), \\
S_{\triangleleft}=\left(\begin{array}{cc}
i S_{\operatorname{cosp}} & 0 \\
0 & i S_{\mathrm{sp}}
\end{array}\right) .
\end{gathered}
$$

Then it holds that

$$
D_{\triangleleft} D_{\triangleright}=D_{\triangleright} D_{\triangleleft}=P_{\text {double }}=\left(\begin{array}{cc}
P_{\text {cosp }} & 0 \\
0 & P_{\mathrm{sp}}
\end{array}\right)
$$

where $P_{\text {... }}$ denotes the Lichnerowicz wave operator on spinors and cospinors, respectively; moreover, one finds that $\Gamma$ commutes with $P_{\text {double }}$ and

$$
\begin{aligned}
\Gamma D_{\triangleleft} & =-D_{\triangleleft} \Gamma, \quad \Gamma D_{\triangleright}=-D_{\triangleright} \Gamma \\
\left(D_{\triangleleft} F_{1}, F_{2}\right) & =-\left(F_{1}, D_{\triangleleft} F_{2}\right), \quad\left(D_{\triangleright}, F_{1}, F_{2}\right)=-\left(F_{1}, D_{\triangleright} F_{2}\right) \quad \forall F_{1}, F_{2} \in \mathscr{D}_{\text {double }} .
\end{aligned}
$$

One may also check that $S_{\triangleleft}^{ \pm}$(defined in obvious analogy to $\left.S_{\triangleleft}\right)$ are the retarded(+)/ad$\operatorname{vanced}(-)$ fundamental solutions for the operator $D_{\triangleright}$; consequently

$$
D_{\triangleright} S_{\triangleleft}=S_{\triangleleft} D_{\triangleright}=0 .
$$

Furthermore, from (2.31) one can see that

$$
\Gamma S_{\triangleleft}=-S_{\triangleleft} \Gamma
$$

This entails that $\Gamma$ is a complex conjugation for the sesquilinear form

$$
\left(F_{1}, F_{2}\right)_{S}=\left(S_{\triangleleft} F_{1}, F_{2}\right), \quad F_{1}, F_{2} \in \mathscr{D}_{\text {double }},
$$

so that

$$
\left(\Gamma F_{1}, \Gamma F_{2}\right)_{S}=\left(F_{2}, F_{1}\right)_{S} \quad \forall F_{1}, F_{2} \in \mathscr{D}_{\text {double }}
$$


On the other hand one can see that (cf. (2.25))

$$
\left(\left[\begin{array}{c}
h_{1} \\
f_{1}
\end{array}\right],\left[\begin{array}{c}
h_{2} \\
f_{2}
\end{array}\right]\right)_{S}=-i\left\langle f_{1}^{+}, S_{\mathrm{sp}} f_{2}\right\rangle+i\left\langle S_{\mathrm{cosp}} h_{2}, h_{1}^{+}\right\rangle
$$

and this implies by Prop. 2.2 in [5] that $(., .)_{S}$ is positive-semidefinite, $(F, F)_{S} \geq 0$.

Now we introduce the quotient space $\mathscr{D}_{\text {double }} / \operatorname{ker} S_{\triangleleft}$ and denote by $\mathscr{H}$ its completion with respect to $(., .)_{S}$. The conjugation $\Gamma$ induces by (2.34) a conjugation on $\mathscr{H}$ which will again be denoted by $\Gamma$. Hence, we have derived from the doubled Dirac equation a complex Hilbert-space $\mathscr{H}$ (with scalar product $(., .)_{S}$ ) together with a complex conjugation $\Gamma$. The system can be quantized, following Araki [1], by assigning to these data the algebra of canonical anti-commutation relations $\operatorname{CAR}(\mathscr{H}, \Gamma)$. This is the unique $C^{*}$-algebra with unit $\mathbb{1}$ which is generated by a family $\{B(\mathbf{v}): \mathbf{v} \in \mathscr{H}\}$ subject to the relations:

(i) $\mathbf{v} \mapsto B(\mathbf{v})$ is $\mathbb{C}$-linear,

(ii) $B(\Gamma \mathbf{v})=B(\mathbf{v})^{*}$,

(iii) $B(\mathbf{v})^{*} B(\mathbf{w})+B(\mathbf{w}) B(\mathbf{v})^{*}=(\mathbf{v}, \mathbf{w})_{S} \cdot \mathbb{1}$.

Now let $q: \mathscr{D}_{\text {double }} \rightarrow \mathscr{D}_{\text {double }} / \operatorname{ker} S_{\triangleleft}$ denote the quotient map, then we define the quantized Dirac field as the linear map which assigns to each $h \in \mathscr{D}_{\text {cosp }}$ the element

$$
\Psi(h)=B\left(q\left[\begin{array}{l}
h \\
0
\end{array}\right]\right)
$$

in $\operatorname{CAR}(\mathscr{H}, \Gamma)$. The adjoint spinor field will be defined by

$$
\Psi^{+}(f)=B\left(q\left[\begin{array}{l}
0 \\
f
\end{array}\right]\right), \quad f \in \mathscr{D}_{\mathrm{sp}} .
$$

As a consequence of (iii), the field and its adjoint satisfy the anti-commutation relations

$$
\Psi(h) \Psi^{+}(f)+\Psi^{+}(f) \Psi(h)=-i\left\langle h, S_{\mathrm{sp}} f\right\rangle .
$$

We also note that, owing to $(2.33), B\left(q\left(D_{\triangleright} F\right)\right)=0$, and this entails

$$
\Psi((i \nabla \nabla+m) h)=0 \quad \text { and } \quad \Psi^{+}((-i \not \nabla+m) f)=0
$$

for all $h \in \mathscr{D}_{\text {cosp }}$ and $f \in \mathscr{D}_{\mathrm{sp}}$.

Remarks. (i) $\Psi$ acts linearly on cospinors and fulfills, in the sense of distributions, the equation $(-i \nabla \nabla+m) \Psi=0$, therefore the map $h \mapsto \Psi(h)$ is regarded as a spinor field. Similarly, $\Psi^{+}$acts linearly on spinor fields and fulfills in distributional sense $(i \not \nabla+m) \Psi^{+}=$ 0 ; hence it is viewed as a cospinor field.

(ii) $\Psi$ and $\Psi^{+}$are $C^{*}$-valued distributions since e.g. $2\left\|\Psi^{+}(f)\right\|^{2}=-i\left\langle f^{+}, S_{\mathrm{sp}} f\right\rangle$ and $\mathscr{D}_{\mathrm{sp}} \otimes \mathscr{D}_{\mathrm{sp}} \ni f_{1} \otimes f_{2} \mapsto-i\left\langle f_{1}^{+}, S_{\mathrm{sp}} f_{2}\right\rangle$ is continuous (with respect to the usual test-function topology). 
(iii) We briefly comment on the case where $\gamma_{0}, \ldots, \gamma_{3}$ belong to a Majorana representation (as considered in [38]) and one wishes to quantize the Majorana field. In that situation, $\mathscr{D}_{\text {cosp }}$ (and similarly, $\mathscr{D}_{\mathrm{sp}}$ ) carries an 'intrinsic' charge conjugation $\Gamma$ given by $v=v_{B} E^{B} \mapsto$ $\overline{v_{B}} E^{B}$ in any frame. Then $\Gamma$ is a skew-conjugation for the sesquilinear form

$$
\left(h, h^{\prime}\right)=\int_{M} d \mu_{\boldsymbol{g}}(p) h_{p}^{\prime}\left(h_{p}^{+}\right)
$$

on $\mathscr{D}_{\text {cosp. }}$ Upon defining $D_{\triangleright}=\not \nabla-i m, D_{\triangleleft}=\not \nabla+i m$ and $S_{\triangleleft}=i S_{\text {cosp }}$, one obtains similar relations as before. Then $\mathscr{H}$ arises as completion of $\mathscr{D}_{\text {cosp }} / \operatorname{ker} S_{\triangleleft}$ and $\left(h, h^{\prime}\right)_{S}=\left(S_{\triangleleft} h, h^{\prime}\right)$. The field operators then simplify to

$$
\Psi(h)=B(q(h)) ; \quad \Psi^{+}(f)=\Psi\left(f^{+}\right)^{*}=\Psi\left(\Gamma f^{+}\right)
$$

for $h \in \mathscr{D}_{\text {cosp }}, f \in \mathscr{D}_{\text {sp }}$. In other words, the Majorana field may be quantized without doubling the classical system. Instead of starting with $\mathscr{D}_{\text {cosp }}$ one can likewise consider $\mathscr{D}_{\text {sp }}$ this has been done in [38.

\subsection{Hadamard states}

We recall that a state on a $C^{*}$-algebra $\mathcal{C}$ is a linear functional $\omega: \mathcal{C} \rightarrow \mathbb{C}$ fulfilling $\omega(\mathbb{1})=1$ and $\omega\left(A^{*} A\right) \geq 0$ for all $A \in \mathcal{C}$. For the purpose of the present work, it is sufficient to focus on the two-point functions $\omega_{2}$ of states $\omega$ on $\operatorname{CAR}(\mathscr{H}, \Gamma)$. The two-point function $\omega_{2}$ of $\omega$ is an element in $\left(\mathscr{D}_{\text {double }} \otimes \mathscr{D}_{\text {double }}\right)^{\prime}$ given by

$$
\omega_{2}\left(F_{1} \otimes F_{2}\right)=\omega\left(B\left(q\left(F_{1}\right)\right) B\left(q\left(F_{2}\right)\right)\right), \quad F_{1}, F_{2} \in \mathscr{D}_{\text {double }} .
$$

It was shown in [1] that there is for each state $\omega$ on $\operatorname{CAR}(\mathscr{H}, \Gamma)$ a linear operator $Q$ on $\mathscr{H}$ with the properties:

(I) $0 \leq Q^{*}=Q \leq 1$,

(II) $Q+\Gamma Q \Gamma=\mathbb{1}$,

(III) $\omega_{2}\left(F_{1} \otimes F_{2}\right)=\left(\Gamma q\left(F_{1}\right), Q q\left(F_{2}\right)\right)_{S} \quad$ for all $F_{1}, F_{2} \in \mathscr{D}_{\text {double }}$.

Conversely, each linear operator $Q$ with the properties (I) and (II) determines by (III) the two-point function $\omega_{2}$ of some state $\omega$ on $\operatorname{CAR}(\mathscr{H}, \Gamma)$, a so-called quasifree state, determined by the two-point function $\omega_{2}$ (see [1] for discussion). Such a quasifree state $\omega$ is pure (and then often called a Fock-state) if and only if $Q$ is a projection, i.e. $Q^{2}=Q$. The operator $Q$ with the properties (I), (II) and (III) above will be called the operator labelling the quasifree state $\omega$.

The stress-energy tensor is defined using the two-point functions of a particular class of states, the Hadamard states. One says that a state $\omega$ on $\operatorname{CAR}(\mathscr{H}, \Gamma)$ is a Hadamard state if we may write

$$
\omega_{2}\left(F_{1} \otimes F_{2}\right)=w\left(D_{\triangleleft} F_{1} \otimes F_{2}\right)
$$


for some distribution $w \in\left(\mathscr{D}_{\text {double }} \otimes \mathscr{D}_{\text {double }}\right)^{\prime}$ of Hadamard form for the doubled waveoperator $P_{\text {double }}$ on $\mathscr{D}_{\text {double }}$; the definition of a Hadamard form for such a wave-operator has been given in [38] (cf. also [28, 29, 23, 30]). This definition entails that the difference between the two-point functions of two Hadamard states is smooth. For the purposes of this work, we will also need the characterization of Hadamard states in terms of properties of the wave-front set $\mathrm{WF}\left(\omega_{2}\right)$ that appears in [29, 23, 38], following a line of argument given in 35 for the scalar case. The relevant statement, proven in the references just stated, is: A state $\omega$ on $\operatorname{CAR}(\mathscr{H}, \Gamma)$ is a Hadamard state if and only if the wave-front set of its two-point function $\omega_{2}$ satisfies the relation

$$
\mathrm{WF}\left(\omega_{2}\right)=\left\{\left(p, \xi ; p^{\prime},-\xi^{\prime}\right) \in \dot{T}^{*}(M \times M) \mid(p, \xi) \sim\left(p^{\prime}, \xi^{\prime}\right) ; \quad \xi \in \mathcal{N}_{p}^{+}\right\},
$$

where $\dot{T}^{*}(M \times M)$ is the cotangent bundle over $M \times M$ without the zero-section, $(p, \xi) \sim$ $\left(p^{\prime}, \xi^{\prime}\right)$ means that there is a lightlike geodesic connecting the points $p$ and $p^{\prime}$ in $M$ and to which $\xi$ and $\xi^{\prime}$ are co-tangent, and $\mathcal{N}_{p}^{+}$is the set of all future-directed null covectors at $p$. (We remark that in 38 the opposite sign convention for the Fourier transform was chosen, leading to the opposite constraint $\xi \in \mathcal{N}_{p}^{-}$(i.e., past-directed null covectors) in that reference compared to (2.46).)

At this point we very briefly recall the definition of the wave-front set of a distribution [24]. For a distribution $t \in \mathscr{D}^{\prime}\left(\mathbb{R}^{n}\right)$, a point $(x, k) \in \mathbb{R}^{n} \times\left(\mathbb{R}^{n} \backslash\{0\}\right)$ is called a regular directed point of $t$ if there exists $\chi \in \mathscr{D}\left(\mathbb{R}^{n}\right)$ with $\chi(x) \neq 0$ and a conic open neighbourhood $C$ of $k$ in $\mathbb{R}^{n} \backslash\{0\}$ such that

$$
\sup _{k^{\prime} \in C}\left(1+\left|k^{\prime}\right|\right)^{N}\left|\widehat{\chi} t\left(k^{\prime}\right)\right|<\infty \quad \forall N \in \mathbb{N} .
$$

(If this holds we will say that $\widehat{\chi} t$ is of rapid decay in $C$.) The complement in $\mathbb{R}^{n} \times\left(\mathbb{R}^{n} \backslash\{0\}\right)$ of the set of all regular directed points of $t$ is called the wave-front set WF $(t)$ of $t$. Given a scalar distribution $\tau$ on a manifold $M$, one says that a non-zero covector $(p, \xi) \in \dot{T}^{*}(M)$ is in $\mathrm{WF}(\tau)$ if there is a coordinate chart $(U, \kappa)$ around $p \in M$ so that $\left(\kappa(p),{ }^{t}\left(\kappa^{-1}\right)^{\prime} \xi\right) \in$ WF $\left(\tau \circ \kappa^{-1}\right)$ where (as discussed at the end of Sec. 目) $\tau \circ \kappa^{-1} \in \mathscr{D}^{\prime}(\kappa(U))$ is a distribution on the chart range of $\kappa$. This definition of $\mathrm{WF}(\tau)$ is independent of the choice of the chart $\kappa$. We refer the reader to [24] for further discussion of the properties of wave-front set of distributions on manifolds. For the case that $\tau$ is a distribution on test-sections of a vector bundle, e.g. defined on $\mathscr{D}_{\text {double, }} \tau$ can be viewed, via (local) trivializations of the bundle, as collection $\left(\tau_{B}^{A}\right)_{A, B}$ of scalar distributions, and then WF $(\tau)$ is defined as the union of $\mathrm{WF}\left(\tau^{A}{ }_{B}\right)$ over all components $A, B$. It is not difficult to see that this definition is independent of the chosen (local) trivialization.

Now let $\omega_{2}$ be the two-point function of a Hadamard state $\omega$ on $\mathrm{CAR}(\mathscr{H}, \Gamma)$, and let $Q$ be the corresponding operator on $\mathscr{H}$ with the properties (I),(II) and (III) above. We will use the notation

$$
Q^{\Gamma}=\Gamma Q \Gamma
$$

for the 'charge conjugate' of $Q$, and we will adopt this notation also for other operators on $\mathscr{H}$. In studying the stress-energy tensor, we will be particularly interested in the 
following distributions on $\mathscr{D}_{\text {sp }} \otimes \mathscr{D}_{\text {cosp }}$ associated with $\omega$ (respectively, with $Q$ ), which we will also refer to as two-point functions:

$$
\omega_{Q}(f \otimes h)=\omega\left(\Psi^{+}(f) \Psi(h)\right), \quad \omega_{Q}^{\Gamma}(f \otimes h)=\omega\left(\Psi(h) \Psi^{+}(f)\right)
$$

where $f \in \mathscr{D}_{\mathrm{sp}}$ and $h \in \mathscr{D}_{\text {cosp. }}$. Note that $\omega_{Q}^{\Gamma}=\omega_{Q^{\Gamma}}$. As a consequence of the constraint on the wave-front set (2.46) for Hadamard states, which is also called microlocal spectrum condition, one finds that the following microlocal spectrum condition holds for $\omega_{Q}$ and $\omega_{Q}^{\Gamma}$,

$$
\mathrm{WF}\left(\omega_{Q}^{\sharp}\right)=\left\{\left(p, \xi ; p^{\prime},-\xi^{\prime}\right) \in \dot{T}^{*}(M \times M) \mid(p, \xi) \sim\left(p^{\prime}, \xi^{\prime}\right) ; \quad \xi \in \mathcal{N}_{p}^{\sharp}\right\} .
$$

Here, and below, we use $\sharp$ and $b$ to denote either the presence or absence of a $\Gamma$ in the following context-dependent way:

\begin{tabular}{cccc}
$Q^{\sharp}$ & $\omega_{Q}^{\sharp}$ & $\mathcal{N}_{p}^{\sharp}$ & $\mathbb{R}^{\sharp}$ \\
\hline \hline$Q$ & $\omega_{Q}$ & $\mathcal{N}_{p}^{+}$ & $\mathbb{R}^{+}$ \\
$Q^{\Gamma}$ & $\omega_{Q}^{\Gamma}$ & $\mathcal{N}_{p}^{-}$ & $\mathbb{R}^{-}$
\end{tabular}.

To avoid confusion we will sometimes use $\cdot$ as a placeholder to indicate the absence of a $\Gamma$. Thus $Q=Q$, for example. We note that the microlocal spectrum condition in the form (2.50) has been proved directly for quasifree Hadamard states in 29, 23.

Let us note that 2.49) may be written

$$
\omega_{Q}(f \otimes h)=\left(\Gamma q\left[\begin{array}{l}
0 \\
f
\end{array}\right], Q q\left[\begin{array}{l}
h \\
0
\end{array}\right]\right)_{S} ;
$$

as a slight abuse of notation we will use this relation to define $\omega_{Q}$ for general bounded operators $Q$ on $\mathscr{H}$ and refer to $\omega_{Q}$ as the two-point function labelled by $Q$. (Of course $\omega_{Q}$ is not in general the two-point function of a state.) We will also denote by $\operatorname{Had}(\mathscr{H}, \Gamma)$ the class of operators which obey properties (I) and (II) and such that $\omega_{Q}^{\sharp}$ obeys (2.50). Thus $\operatorname{Had}(\mathscr{H}, \Gamma)$ parametrizes the quasifree Hadamard states on $\mathrm{CAR}(\mathscr{H}, \Gamma)$.

Our proof of Thm. 4.1 below relies on the existence of pure, quasifree Hadamard states for the Dirac field. It seems that this has never been established in the literature in full detail, therefore we sketch here how such states may be constructed by adapting an argument employed by Fulling, Narcowich and Wald [19] for the case of the free scalar field to the Dirac field. The first step is to show that there exists a pure, quasifree Hadamard state for the Dirac field for ultrastatic $(M, \boldsymbol{g})$. In fact, if $(M, \boldsymbol{g})$ is ultrastatic, then it may be endowed with a suitable spin structure so that the ultrastatic time shifts give rise to a continuous unitary group $U_{t}(t \in \mathbb{R})$ on $\mathscr{H}$ leaving the scalar product $(., .)_{S}$ invariant and fulfilling $\Gamma U_{t}=U_{t} \Gamma$. Moreover, if the mass parameter $m$ appearing in the Dirac equation is strictly positive, then the spectrum of the self-adjoint generator of $U_{t}$ is bounded away from zero. Theorem 2 in [1], or the results in [47, thus show that there is a pure quasifree state $\omega_{0}$ on the CAR algebra of the Dirac field on ultrastatic $(M, \boldsymbol{g})$ which is a ground state for the $C^{*}$-dynamics induced by $U_{t}$; the projection $P_{0}$ labelling $\omega_{0}$ is the projection onto the positive spectral subspace of the unitary group $U_{t}$. 
Since $\omega_{0}$ is a ground state, it fulfills the microlocal spectrum condition [37] and hence is a Hadamard state [38. In a second step, one uses a technique developed in [19] which allows one to view a neighbourhood of a Cauchy surface of any given globally hyperbolic spacetime as being isometrically embedded in a globally hyperbolic spacetime that has an ultrastatic part (with suitable spin structure as above) in its past. By the uniqueness of the Cauchy problem and the "propagation of Hadamard form" under the dynamics of the Dirac equation [38], any pure, quasifree Hadamard state prescribed on the ultrastatic part of the spacetime (e.g. $\left.\omega_{0}\right)$ induces a pure, quasifree Hadamard state everywhere on the spacetime, in particular on the embedded neighbourhood of the Cauchy surface of the initially given globally hyperbolic spacetime. Using the same argument once more, a pure, quasifree Hadamard state for the Dirac field is thereby induced on any given globally hyperbolic spacetime. The mass parameter $m$ may be allowed to be variable over spacetime in this process without affecting the Hadamard form, so that one obtains a pure, quasifree Hadamard state of the Dirac field on any globally hyperbolic spacetime for any $m \geq 0$. The argument just sketched implicitly also shows that there exists an abundance of quasifree Hadamard states.

\section{A point-split energy density}

For the remainder of this paper, we will assume that $(M, \boldsymbol{g})$ is globally hyperbolic, orientable and time orientable, with spin structure $(S(M, \boldsymbol{g}), \psi)$ and that the Dirac matrices $\gamma_{a}$ belong to a standard representation.

Let $\gamma: \mathbb{R} \rightarrow M$ be a smooth timelike curve in $(M, \boldsymbol{g})$, parametrized by its proper time, along which we wish to establish a QWEI. The starting point is the construction of a normal ordered energy density on $\gamma$, which is accomplished as follows. We first claim that there exists a tubular neighbourhood $C_{\gamma}$ of $\gamma$ and a local section $E$ of $S(M, \boldsymbol{g})$ over $C_{\gamma}$ such that the induced tetrad field $\left(e_{0}, \ldots, e_{3}\right)=\psi \circ E$ satisfies $\left.e_{0}\right|_{\gamma}=u$, where $u=\dot{\gamma}$ is the velocity of $\gamma$. To see this, choose any locally finite open cover $\left\{U_{j} \mid j \in \mathbb{Z}\right\}$ of $\gamma$ by charts $U_{j}$ such that

(i). $U_{j} \cap U_{k}=\emptyset$ unless $|j-k| \leq 1$;

(ii). $U_{j} \cap U_{j+1}$ is contractable for each $j \in \mathbb{Z}$;

(iii). $U_{j} \cap U_{k} \cap U_{l}=\emptyset$ if $j, k, l$ are distinct.

The existence of such a cover follows from global hyperbolicity of $(M, \boldsymbol{g})$ since $\gamma$ is timelike. Now extend $u$ to a smooth timelike unit vector field $\widetilde{u}$ on some tubular neighbourhood $C_{\gamma}$ of $\gamma$, so that $C_{\gamma} \subset \bigcup_{j} U_{j}$. Choose any tetrad $\left(e_{0}^{\prime}, \ldots, e_{3}^{\prime}\right)$ on $C_{\gamma}$. Then we may obtain a tetrad $\left(e_{0}, \ldots, e_{3}\right)$ with $e_{0}=\widetilde{u}$ by applying a unique boost in $\mathscr{L}_{+}^{\uparrow}$ at each point (whose parameters are given by the components of $\widetilde{u}$ with respect to $e_{a}^{\prime}$, and therefore vary smoothly). This tetrad lifts smoothly to $S(M, \boldsymbol{g})$ in each $U_{j} \cap C_{\gamma}$ and may be patched together along $C_{\gamma}$ to obtain the required section $E$ by virtue of properties (i), (ii) and (iii). [

\footnotetext{
${ }^{5}$ Of course, there are exactly two such sections.
} 
Next, we choose smooth spinor fields $v_{A}(A=1, \ldots, 4)$ in $C_{\gamma}$, such that

$$
\delta^{A B} v_{A} \otimes v_{B}^{+}=\gamma_{0} .
$$

This is easily satisfied by taking $v_{A}=E_{A}$, where $E_{A}$ is the spin frame induced by $E$; however, it will be convenient to make a slightly different choice when considering the Majorana field. The changes relevant for Majorana fields will be described in Sec. 7 .

With respect to the frame $e_{a}$ the Dirac stress-energy tensor is

$$
T_{a b}=\frac{i}{2}\left(\psi^{+} \gamma_{(a} \nabla_{b)} \psi-\left(\nabla_{(a} \psi^{+}\right) \gamma_{b)} \psi\right),
$$

which is manifestly symmetric, and is conserved provided $\psi$ obeys the Dirac equation (2.17). In particular, $T_{00}(\gamma(\tau))$ is the energy density measured by an observer with worldline $\gamma$ at proper time $\tau$.

We may use (3.1) to define a bi-scalar point-split energy density

$$
\mathcal{T}(x, y)=\delta^{A B} \frac{i}{2}\left(\left(\psi^{+} v_{A}\right)(x)\left(v_{B}^{+} e_{0} \cdot \nabla \psi\right)(y)-\left(\left[e_{0} \cdot \nabla \psi^{+}\right] v_{A}\right)(x)\left(v_{B}^{+} \psi\right)(y)\right)
$$

with the property that $\mathcal{T}(x, x)=T_{00}(x)$. Integrating by parts, $\mathcal{T}$ becomes a scalar bidistribution $\mathcal{T} \in(\mathscr{D}(M) \otimes \mathscr{D}(M))^{\prime}$

$$
\mathcal{T}(f \otimes g)=\delta^{A B} \frac{i}{2}\left(\psi^{+}\left(\nabla \cdot\left[e_{0} v_{A} f\right]\right) \psi\left(v_{B}^{+} g\right)-\psi^{+}\left(v_{A} f\right) \psi\left(\nabla \cdot\left[e_{0} v_{B}^{+} g\right]\right)\right) .
$$

Here (and below) the notation $\nabla \cdot\left[e_{0} v\right]$ denotes minus the distributional dual of $e_{0} \cdot \nabla$, applied to the test function or (co)spinor $v$. Thus,

$$
\nabla \cdot\left[e_{0} v\right]=v \nabla \cdot e_{0}+e_{0} \cdot \nabla v
$$

where, with respect to local coordinates $\left(x^{\mu}\right), \nabla \cdot e_{0}=\nabla_{\mu} e_{0}^{\mu}$ and $e_{0} \cdot \nabla v=e_{0}^{\mu} \nabla_{\mu} v$. Upon quantization, we obtain the algebra-valued bi-distribution $T$ given by

$$
T(f \otimes g)=\delta^{A B} \frac{i}{2}\left(\Psi^{+}\left(\nabla \cdot\left[e_{0} v_{A} f\right]\right) \Psi\left(v_{B}^{+} g\right)-\Psi^{+}\left(v_{A} f\right) \Psi\left(\nabla \cdot\left[e_{0} v_{B}^{+} g\right]\right)\right) .
$$

Given a state $\omega$ we will now use the same symbol to denote its two-point function $\omega(f \otimes g)=\omega\left(\Psi^{+}(f) \Psi(g)\right)$ and also set $v_{A B}=v_{A} \otimes v_{B}^{+}$. Thus $v_{A B} \omega$ will denote the matrix of scalar bi-distributions

$$
v_{A B} \omega(f \otimes g)=\omega\left(\Psi^{+}\left(v_{A} f\right) \Psi\left(v_{B}^{+} g\right)\right) .
$$

The formulae $\nabla \cdot\left(e_{0} v_{A} f\right)=v_{A} \nabla \cdot\left(e_{0} f\right)+\sigma_{0}{ }_{A}{ }_{A} f v_{C}$ and $\nabla \cdot\left(e_{0} v_{B}^{+} g\right)=v_{B}^{+} \nabla \cdot\left(e_{0} g\right)+\overline{\sigma_{0}{ }_{B}} g v_{C}^{+}$ now allow us to write the expectation value $\langle T\rangle_{\omega}$ of $T$ in state $\omega$ as

$$
\langle T\rangle_{\omega}=\mathcal{L}^{A B} v_{A B} \omega,
$$

where

$$
\mathcal{L}^{A B}=\frac{1}{2}\left(\mathbb{1} \otimes i e_{0} \cdot \nabla-i e_{0} \cdot \nabla \otimes \mathbb{1}\right) \delta^{A B}+\frac{1}{2} \Theta^{A B},
$$


and

$$
\Theta^{A B}=i\left[\delta^{C B} \sigma_{0}{ }^{A} C \otimes \mathbb{1}-\mathbb{1} \otimes \delta^{A C} \overline{\sigma_{0}{ }^{B} C}\right]
$$

If a reference Hadamard state $\omega_{0}$ is now specified, we may define the normal ordered point-split energy density (with respect to $\omega_{0}$ ) by

$$
\langle: T:\rangle_{\omega}=\langle T\rangle_{\omega}-\langle T\rangle_{\omega_{0}}
$$

This may also be written

$$
\langle: T:\rangle_{\omega}=\mathcal{L}^{A B} v_{A B}: \omega:
$$

where : $\omega:=\omega-\omega_{0}$ is the normal ordered two-point function. Since : $\omega:$ is smooth for Hadamard $\omega,\langle: T:\rangle_{\omega}$ is also smooth. Accordingly, the 'coincidence limit' (i.e., the restriction of $\langle: T:\rangle_{\omega}$ to the diagonal) is well defined and yields the normal ordered energy density near $\gamma$. We denote the energy density along $\gamma$ by

$$
\langle: \rho:\rangle_{\omega}(\tau)=\langle: T:\rangle_{\omega}(\gamma(\tau), \gamma(\tau))
$$

Note also that $\langle: T:\rangle_{\omega}(f \otimes g)$ is symmetric in $f$ and $g$ by virtue of the CAR's.

It will be convenient to regard $\langle: \rho:\rangle_{\omega}$ as the diagonal of the pull-back $\gamma_{2}^{*}\langle: T:\rangle_{\omega}$ where $\gamma_{2}\left(\tau, \tau^{\prime}\right)=\left(\gamma(\tau), \gamma\left(\tau^{\prime}\right)\right)$. In turn, $\gamma_{2}^{*}\langle: T:\rangle_{\omega}$ may be written as the action of a differential operator on the pulled-back normal ordered two-point function:

$$
\gamma_{2}^{*}\langle: T:\rangle_{\omega}=\frac{1}{2}\left[(\mathbb{1} \otimes D-D \otimes \mathbb{1}) \delta^{A B}+\gamma_{2}^{*} \Theta^{A B}\right] \gamma_{2}^{*} v_{A B}: \omega:,
$$

where $D=i d / d \tau$ (strictly speaking, $D$ should be regarded as the distributional dual of $-i d / d \tau)$.

\section{Main argument}

We now come to the proof of the QWEI for Dirac fields. In the following, $(M, \boldsymbol{g})$ is assumed to satisfy the hypotheses stated at the beginning of the previous section.

Theorem 4.1 Let $\gamma: \mathbb{R} \rightarrow M$ be a smooth timelike curve in $(M, \boldsymbol{g})$ parametrized by its proper time. Let $\omega_{0}$ be a Hadamard state of the Dirac field on $(M, \boldsymbol{g})$. Define the normal ordered energy density $\langle: \rho:\rangle_{\omega}$ by (3.13) with respect to the reference state $\omega_{0}$. Then for any weight $f$ belonging to $\mathscr{W}$ (defined in Eq. (1.1))

$$
\inf _{\omega} \int d \tau\langle: \rho:\rangle_{\omega}(\tau) f(\tau)>-\infty
$$

where the infimum is taken over all Hadamard states $\omega$. That is, there exists a quantum weak energy inequality for the Dirac field. 
Remarks. (i) If the reference state $\omega_{0}$ is changed, $\langle: \rho:\rangle_{\omega}$ is modified by a smooth function which is independent of $\omega$. Thus we may assume without loss of generality that $\omega_{0}$ is pure and quasifree. Exactly the same argument entails that (4.1) holds if we replace the normal ordered energy density by the renormalised energy density.

(ii) Perhaps surprisingly, the class $\mathscr{W}$ (of squares of real-valued $C_{0}^{\infty}(\mathbb{R})$ functions) does not coincide with the class of nonnegative smooth compactly supported functions. In fact, Glaeser [20 [ has constructed an example of a $C^{\infty}$ nonnegative function $f$, vanishing only at the origin, so that $\frac{d^{2}}{d x^{2}} \sqrt{f(x)}$ diverges as $x \rightarrow 0$. The delicacy of this point resides in the behaviour of $f$ at zeros of infinite order. It is not clear whether the restriction to weights in $\mathscr{W}$ is purely a technical limitation of our proof, or whether QWEIs should be understood as quadratic form results (cf. [10]).

Proof: It is sufficient to prove (4.1) for arbitrary $f \in C_{0}^{\infty}(I) \cap \mathscr{W}$ where $I \subset \mathbb{R}$ is an arbitrary open interval with compact closure. To this end, choose $\eta \in C_{0}^{\infty}(M)$ such that $\eta$ equals unity on a neighbourhood of $\gamma(I)$. It is easy to see that $\langle: \rho:\rangle_{\omega}$ is unaltered on $I$ if we replace $v_{A B}: \omega:$ in (3.14) by the compactly supported distribution $u_{A B}: \omega:$, where

$$
u_{A B}=\eta v_{A} \otimes \eta v_{B}^{+} .
$$

Applying the formula

$$
\int d \tau F(\tau, \tau) \varphi(\tau)=\int \frac{d \lambda d \lambda^{\prime}}{(2 \pi)^{2}} \widehat{F}\left(-\lambda, \lambda^{\prime}\right) \widehat{\varphi}\left(\lambda-\lambda^{\prime}\right)
$$

which is valid for $F \in C_{0}^{\infty}\left(\mathbb{R}^{2}\right)$ and $\varphi \in C_{0}^{\infty}(\mathbb{R})$, one may show that

$$
\mathcal{I}=\int d \tau\langle: \rho:\rangle_{\omega}(\tau) f(\tau)=\int d \lambda d \lambda^{\prime} J^{A B}\left(\lambda, \lambda^{\prime}\right) W_{A B}^{(\omega)}\left(\lambda, \lambda^{\prime}\right)
$$

where

$$
W_{A B}^{(\omega)}\left(\lambda, \lambda^{\prime}\right)=\left[\gamma_{2}^{*} u_{A B}: \omega:\right]^{\wedge}\left(-\lambda, \lambda^{\prime}\right)
$$

and we have also written

$$
J^{A B}\left(\lambda, \lambda^{\prime}\right)=\frac{1}{8 \pi^{2}}\left\{\left(\lambda+\lambda^{\prime}\right) \widehat{f}\left(\lambda-\lambda^{\prime}\right) \delta^{A B}+\left[\theta^{A B} f\right]^{\wedge}\left(\lambda-\lambda^{\prime}\right)\right\}
$$

where

$$
\theta^{A B}(\tau)=\gamma_{2}^{*} \Theta^{A B}(\tau, \tau)=i\left(\left.\delta^{C B} \sigma_{0}^{A}{ }_{C}\right|_{\gamma(\tau)}-\delta^{A C} \overline{\left.\sigma_{0}^{B}{ }_{C}\right|_{\gamma(\tau)}}\right)
$$

is clearly hermitian $\left.\overline{\left(\theta^{B A}(\tau)\right.}=\theta^{A B}(\tau)\right)$. It follows that $J^{A B}$ is a hermitian matrix kernel, i.e., $J^{A B}\left(\lambda, \lambda^{\prime}\right)=\overline{J^{B A}\left(\lambda^{\prime}, \lambda\right)}$.

Note that $J^{A B}$ is state-independent, while $W_{A B}^{(\omega)}$ contains all the dependence on the state of interest $\omega$ and the reference state $\omega_{0}$. We also note that $J^{A B}\left(\lambda, \lambda^{\prime}\right)$ decays rapidly away from the diagonal in $\mathbb{R}^{2}$.

\footnotetext{
${ }^{6}$ We are grateful to S.P. Eveson and P.J. Bushell for help in locating this reference.
} 
Assuming without loss that $\omega_{0}$ is pure and quasifree, it must be labelled by some projection $P$ on $\mathscr{H}$. Since the stress-energy tensor is defined in terms of the two-point function, it is enough to establish (4.1) when the infimum is taken over quasifree Hadamard states, whose two-point functions are of the form $\omega_{Q}$ for $Q \in \operatorname{Had}(\mathscr{H}, \Gamma)$ as discussed in Sec. 2.3. The normal ordered two-point function : $\omega_{Q}:=\omega_{Q}-\omega_{P}$ is labelled by $Q-P$, i.e., $: \omega_{Q}:=\omega_{Q-P}$ in the spirit of the remarks following (2.51). Now

$$
Q-P=-P Q^{\Gamma} P+P^{\Gamma} Q P+P Q P^{\Gamma}+P^{\Gamma} Q P^{\Gamma}
$$

and this induces a decomposition of : $\omega_{Q}$ : into four pieces

$$
: \omega_{Q}:=-\omega^{\cdot \Gamma \cdot}+\omega^{\Gamma \cdot \cdot}+\omega^{\cdot \Gamma}+\omega^{\Gamma \cdot \Gamma},
$$

where $\omega^{\sharp \text { th }}$ is the two-point function labelled by $P^{\sharp} Q^{\natural} P^{b}$; that is,

$$
\omega^{\sharp \natural b}(f \otimes h)=\left(\Gamma q\left[\begin{array}{l}
0 \\
f
\end{array}\right], P^{\sharp} Q^{\natural} P^{b} q\left[\begin{array}{l}
h \\
0
\end{array}\right]\right)_{S}
$$

for $f \in \mathscr{D}_{\text {sp }}$ and $h \in \mathscr{D}_{\text {cosp. }}$. In Sec. 5 we will show that each $\omega^{\sharp \text { th }}$ may be pulled back to $\mathbb{R}^{2}$ by $\gamma_{2}$, allowing us to write

$$
W_{A B}^{\left(\omega_{Q}\right)}=-W_{A B}^{\cdot \Gamma \cdot}+W_{A B}^{\Gamma \ddot{ }}+W_{A B}^{\ddot{\Gamma} \Gamma}+W_{A B}^{\Gamma \cdot \Gamma}
$$

where

$$
W_{A B}^{\sharp \sharp b}\left(\lambda, \lambda^{\prime}\right)=\left[\gamma_{2}^{*} u_{A B} \omega^{\sharp \sharp b}\right]^{\wedge}\left(-\lambda, \lambda^{\prime}\right) .
$$

Furthermore, the following $Q$-independent bounds will be established in Sec. 5 .

Lemma 4.2 For any $Q \in \operatorname{Had}(\mathscr{H}, \Gamma)$,

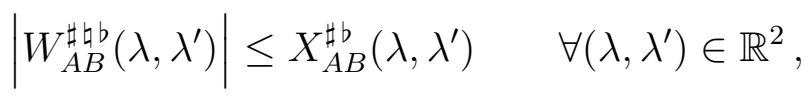

where $X_{A B}$ is independent of $Q$ and is defined in terms of the reference two-point function $\omega_{P}$ by

$$
X_{A B}^{\sharp b}\left(\lambda, \lambda^{\prime}\right)=Y_{A}^{\sharp}(\lambda) Y_{B}^{b}\left(\lambda^{\prime}\right),
$$

where $Y_{A}^{\sharp}(\lambda)$ is the positive square root of

$$
Y_{A}^{\sharp}(\lambda)^{2}=\left[\gamma_{2}^{*} u_{A A} \omega_{P}^{\sharp}\right]^{\wedge}(-\lambda, \lambda) \quad(\text { no sum on } A) \text {. }
$$

Furthermore, $Y_{\dot{A}}(\lambda)$ (resp. $Y_{A}^{\Gamma}(\lambda)$ ) decays rapidly as $\lambda \rightarrow+\infty$ (resp. $\lambda \rightarrow-\infty$ ) and is of polynomially bounded growth as $\lambda \rightarrow-\infty$ (resp. $\lambda \rightarrow+\infty)$. 
Remarks. (i) The right-hand side of Eq. (4.15) is nonnegative because $u_{A A} \omega_{P}^{\sharp}$ is of positive type as a scalar bi-distribution, and this property is inherited under pull-back by $\gamma_{2}$ (see Theorem 2.2 in [8]). In fact, the calculation

$$
u_{A B} \omega_{P}^{\sharp}\left(f^{A} \otimes \overline{f^{B}}\right)=\omega_{P}^{\sharp}\left(f^{A} u_{A} \otimes\left(f^{B} u_{B}\right)^{+}\right)=\omega_{0}\left(\Psi\left(f^{A} u_{A}\right) \Psi\left(f^{B} u_{B}\right)^{*}\right) \geq 0
$$

(summing on $A$ and $B$ ) for $f^{A} \in C_{0}^{\infty}(M)(A=1, \ldots, 4)$ shows that $u_{A B} \omega_{P}^{\sharp}$ is of positive type as a matrix-valued distribution. Positive type of $u_{A A} \omega_{P}^{\sharp}$ follows in consequence. A similar argument (using (4.10) and the property $Q \geq 0$ for $Q \in \operatorname{Had}(\mathscr{H}, \Gamma)$ ) shows that $u_{A B} \omega^{\cdot \Gamma}, u_{A B} \omega^{\Gamma \cdot \Gamma}$ and their pull-backs by $\gamma_{2}$ share the matrix positive type property.

(ii) The statements on the growth of $Y_{A}^{\sharp}$ are obtained from the Paley-Wiener-Schwartz theorem [24] which entails that the Fourier transform of a compactly supported distribution is of at worst polynomial growth. Below, we will frequently use the fact that the product of a rapidly decaying function and one of polynomial growth is itself rapidly decaying.

Because the bounds obtained in Lemma 4.2 exhibit different behaviour in the four quadrants $C_{1}, \ldots, C_{4}$ of the $\left(\lambda, \lambda^{\prime}\right)$-plane it is convenient to decompose the averaged energy density (4.4) as $\mathcal{I}=\sum \mathcal{I}_{k}$, where $\mathcal{I}_{k}$ is the contribution arising from quadrant $C_{k}$. We proceed to bound the $\mathcal{I}_{k}$ in turn.

Starting with the second and fourth quadrants $C_{2}=\mathbb{R}^{-} \times \mathbb{R}^{+}$and $C_{4}=\mathbb{R}^{+} \times \mathbb{R}^{-}$, Lemma 4.2 yields the $Q$-independent bound

$$
\left|W_{A B}^{\left(\omega_{Q}\right)}\left(\lambda, \lambda^{\prime}\right)\right| \leq \sum_{\sharp b} X_{A B}^{\sharp b}\left(\lambda, \lambda^{\prime}\right)
$$

in which each summand on the right-hand side is of at worst polynomial growth. This may be combined with the rapid decay of $J^{A B}$ away from the diagonal to yield the following $Q$-independent bound on the contribution from these quadrants:

$$
\left|\mathcal{I}_{2}+\mathcal{I}_{4}\right| \leq \int_{C_{2} \cup C_{4}} d \lambda d \lambda^{\prime}\left|J^{A B}\left(\lambda, \lambda^{\prime}\right)\right| \sum_{\sharp b} X_{A B}^{\sharp b}\left(\lambda, \lambda^{\prime}\right)<\infty .
$$

We are left with the first and third quadrants. Since $J^{A B}$ exhibits polynomial growth along the diagonal, the previous argument will not allow us to bound all the terms arising from the decomposition (4.11). To see this, note that Lemma 4.2 applied to the $P^{\Gamma} Q P^{\Gamma}$ term $W_{A B}^{\Gamma \cdot \Gamma}$ gives a bound $X_{A B}^{\Gamma}$ growing polynomially $]^{\top}$ in all directions in the first quadrant. Similarly, the bound $X_{A B}$ for the $P Q^{\Gamma} P$ term is polynomially growing in the third quadrant. However, Lemma 4.2 suffices to bound the other contributions to $\mathcal{I}_{1}$ and $\mathcal{I}_{3}$ because at least one factor in the relevant $X_{A B}^{\sharp b}$ is rapidly decaying. Thus

$$
\left|\mathcal{I}_{1}-\mathcal{R}_{1}\right| \leq \int_{C_{1}} d \lambda d \lambda^{\prime}\left|J^{A B}\left(\lambda, \lambda^{\prime}\right)\right|\left[X_{A B}^{\cdot}\left(\lambda, \lambda^{\prime}\right)+X_{A B}^{\cdot \Gamma}\left(\lambda, \lambda^{\prime}\right)+X_{A B}^{\Gamma \cdot}\left(\lambda, \lambda^{\prime}\right)\right]<\infty
$$

and

$$
\left|\mathcal{I}_{3}-\mathcal{R}_{3}\right| \leq \int_{C_{3}} d \lambda d \lambda^{\prime}\left|J^{A B}\left(\lambda, \lambda^{\prime}\right)\right|\left[X_{A B}^{\Gamma \Gamma}\left(\lambda, \lambda^{\prime}\right)+X_{A B}^{\cdot \Gamma}\left(\lambda, \lambda^{\prime}\right)+X_{A B}^{\Gamma \cdot}\left(\lambda, \lambda^{\prime}\right)\right]<\infty
$$

\footnotetext{
${ }^{7}$ Note that it is the bound which is polynomially growing; we expect that $W_{A B}^{\Gamma \cdot \Gamma}$ is actually decaying.
} 
where the remaining terms are

$$
\mathcal{R}_{1}=\int_{C_{1}} d \lambda d \lambda^{\prime} J^{A B}\left(\lambda, \lambda^{\prime}\right) W_{A B}^{\Gamma \cdot \Gamma}\left(\lambda, \lambda^{\prime}\right)
$$

and

$$
\mathcal{R}_{3}=-\int_{C_{3}} d \lambda d \lambda^{\prime} J^{A B}\left(\lambda, \lambda^{\prime}\right) W_{A B}^{\cdot \Gamma \cdot}\left(\lambda, \lambda^{\prime}\right)
$$

(the leading minus sign arises because it is $-P Q^{\Gamma} P$ which appears in (4.8)). The quantities $\mathcal{R}_{1}$ and $\mathcal{R}_{3}$ are real, because $J^{A B}, W_{A B}^{\Gamma \cdot \Gamma}$ and $W_{A B}^{\cdot \Gamma}$ are hermitian matrix kernels.

To complete the proof of the QWEI it is required to show that $\mathcal{R}_{1}$ and $\mathcal{R}_{3}$ are bounded from below independently of $Q$. We will present the argument for $\mathcal{R}_{1}$ in detail and indicate how the proof is modified for $\mathcal{R}_{3}$. Let $\chi_{\Lambda}\left(\Lambda \in \mathbb{R}^{+}\right)$be a family of smooth, real-valued, nonincreasing functions such that $\chi_{\Lambda}$ equals unity on $[0, \Lambda]$ and vanishes on $[\Lambda+1, \infty)$. It is clear that

$$
\mathcal{R}_{1}=\lim _{\Lambda \rightarrow \infty} \int_{C_{1}} d \lambda d \lambda^{\prime} \chi_{\Lambda}(\lambda) \chi_{\Lambda}\left(\lambda^{\prime}\right) J^{A B}\left(\lambda, \lambda^{\prime}\right) W_{A B}^{\Gamma \cdot \Gamma}\left(\lambda, \lambda^{\prime}\right)
$$

and the cut-off $\chi_{\Lambda}$ now allows us to interpret the integral as a trace in the following way. Define $\sigma: \mathbb{R}^{+} \rightarrow \mathbb{R}$ by

$$
\sigma(\lambda)=\left(1+\lambda^{2}\right)^{1 / 2}\left[1+\left|Y^{\Gamma}(\lambda)\right|_{\mathbb{C}^{4}}\right]
$$

where $|\cdot|_{\mathbb{C}^{4}}$ is the usual vector norm on $\mathbb{C}^{4}$. Then $\sigma$ is smooth, positive, bounded away from zero and of polynomially bounded growth. Next, define operators $\widetilde{J}_{\Lambda}$ and $\widetilde{W}$ on $L^{2}\left(\mathbb{R}^{+}, d \lambda\right) \otimes \mathbb{C}^{4}$ by

$$
\left(\widetilde{J}_{\Lambda} \varphi\right)^{A}(\lambda)=\int_{0}^{\infty} d \lambda^{\prime} \sigma_{\Lambda}(\lambda) \sigma_{\Lambda}\left(\lambda^{\prime}\right) J^{A C}\left(\lambda, \lambda^{\prime}\right) \delta_{C B} \varphi^{B}\left(\lambda^{\prime}\right)
$$

and

$$
(\widetilde{W} \varphi)^{B}\left(\lambda^{\prime}\right)=\delta^{B D} \int_{0}^{\infty} d \lambda^{\prime \prime} \frac{W_{C D}^{\Gamma \cdot \Gamma}\left(\lambda^{\prime \prime}, \lambda^{\prime}\right)}{\sigma\left(\lambda^{\prime}\right) \sigma\left(\lambda^{\prime \prime}\right)} \varphi^{C}\left(\lambda^{\prime \prime}\right),
$$

where have written $\sigma_{\Lambda}(\lambda)=\chi_{\Lambda}(\lambda) \sigma(\lambda)$. Now $\widetilde{J}_{\Lambda}$ is Hilbert-Schmidt (due to the cut-off) and self-adjoint while the properties of $\widetilde{W}$ are summarised in the following proposition, which is proved at the end of this section.

Proposition 4.3 For all $Q \in \operatorname{Had}(\mathscr{H}, \Gamma), \widetilde{W}$ is a positive trace-class operator with

$$
0 \leq \operatorname{Tr} \widetilde{W} \leq \frac{\pi}{2}
$$

We may therefore rewrite Eq. (4.23) in the form

$$
\mathcal{R}_{1}=\lim _{\Lambda \rightarrow \infty} \operatorname{Tr} \widetilde{J_{\Lambda}} \widetilde{W}
$$


Introducing an orthornormal basis of eigenvectors $v_{n}$ for $\widetilde{W}$, and using $\widetilde{W} \geq 0$ and (4.27), we have

$$
\begin{aligned}
\operatorname{Tr} \widetilde{J}_{\Lambda} \widetilde{W} & =\sum_{n}\left\langle v_{n} \mid \widetilde{J}_{\Lambda} \widetilde{W} v_{n}\right\rangle=\sum_{n}\left\langle v_{n} \mid \widetilde{J}_{\Lambda} v_{n}\right\rangle\left\langle v_{n} \mid \widetilde{W} v_{n}\right\rangle \\
& \geq \inf \operatorname{spec}\left(\widetilde{J}_{\Lambda}\right) \sum_{n}\left\langle v_{n} \mid \widetilde{W} v_{n}\right\rangle \\
& \geq \inf \operatorname{spec}\left(\widetilde{J}_{\Lambda}\right) \operatorname{Tr} \widetilde{W} \\
& \geq \frac{\pi}{2} \min \left\{0, \inf \operatorname{spec}\left(\widetilde{J}_{\Lambda}\right)\right\} .
\end{aligned}
$$

Noting that the right-hand side has no $Q$-dependence, the required lower bound on $\mathcal{R}_{1}$ now follows from the following proposition, which is proved in Sec. 6.

Proposition 4.4 The spectrum of $\widetilde{J}_{\Lambda}$ is bounded from below uniformly in $\Lambda$ (with a finite lower bound).

Turning to the integral $\mathcal{R}_{3}$, we follow exactly the same argument but with the single difference that the kernel $J^{A B}$ on $\mathbb{R}^{-} \times \mathbb{R}^{-}$defines an operator on $L^{2}\left(\mathbb{R}^{-}\right) \otimes \mathbb{C}^{4}$ which may be bounded above by a nonnegative quantity; this is compensated by the leading minus sign in the definition of $\mathcal{R}_{3}$. Thus $\mathcal{R}_{1}+\mathcal{R}_{3}$ has a finite $Q$-independent lower bound as required and the proof of Theorem 4.1 is complete.

Proof of Proposition 4.3: Using Lemma 4.2 and Cauchy-Schwarz, we first estimate

$$
\left|(\widetilde{W} \varphi)\left(\lambda^{\prime}\right)\right|_{\mathbb{C}^{4}} \leq \frac{\left|Y^{\Gamma}\left(\lambda^{\prime}\right)\right|_{\mathbb{C}^{4}}}{\left(1+\lambda^{\prime 2}\right)^{1 / 2}\left[1+\left|Y^{\Gamma}\left(\lambda^{\prime}\right)\right|_{\mathbb{C}^{4}}\right]} \int d \lambda^{\prime \prime} \frac{Y_{C}^{\Gamma}\left(\lambda^{\prime \prime}\right)}{\left(1+\lambda^{\prime \prime 2}\right)^{1 / 2}\left[1+\left|Y^{\Gamma}\left(\lambda^{\prime \prime}\right)\right|_{\mathbb{C}^{4}}\right]}\left|\varphi^{C}\left(\lambda^{\prime \prime}\right)\right|
$$

for $\varphi \in L^{2}\left(\mathbb{R}^{+}\right) \otimes \mathbb{C}^{4}$, from which we obtain $\|\widetilde{W} \varphi\| \leq \frac{1}{2} \pi\|\varphi\|$. Thus $\widetilde{W}$ is bounded. A short calculation shows that

$$
\langle\varphi \mid \widetilde{W} \varphi\rangle=\left(\gamma_{2}^{*} u_{C B} \omega^{\Gamma \cdot \Gamma}\right)\left(\psi^{C} \otimes \overline{\psi^{B}}\right) \geq 0
$$

for $\varphi \in C_{0}^{\infty}\left(\mathbb{R}^{+}\right) \otimes \mathbb{C}^{4}$ where

$$
\psi(\tau)=\frac{1}{2 \pi}\left[\frac{1}{\sigma} \varphi\right]^{\vee}(\tau) \in \mathscr{S}(\mathbb{R}) \otimes \mathbb{C}^{4},
$$

and we have used the matrix positive type property and compact support of $\gamma_{2}^{*} u_{C B} \omega^{\Gamma \cdot \Gamma}$. Thus $\widetilde{W}$ is positive. In order to show that $\widetilde{W}$ is trace-class it is now enough (by the lemma following Theorem X1.31 in [36]) to show that the formal trace of $\widetilde{W}$ is finite (whereupon the formal trace is indeed the trace). But this is just

$$
\int_{0}^{\infty} d \lambda \sigma(\lambda)^{-2} \delta^{B C} W_{B C}(\lambda, \lambda) \leq \int_{0}^{\infty} d \lambda \frac{\left|Y^{\Gamma}(\lambda)\right|_{\mathbb{C}^{4}}^{2}}{\left(1+\lambda^{2}\right)\left(1+\left|Y^{\Gamma}(\lambda)\right|_{\mathbb{C}^{4}}\right)^{2}} \leq \frac{\pi}{2},
$$

where we have used Lemma 4.2 and the fact that the diagonal of the kernel of a positive trace-class operator is positive. Thus $\widetilde{W}$ is trace-class on $L^{2}\left(\mathbb{R}^{+}\right) \otimes \mathbb{C}^{4}$; putting this together with the positivity property, we have $0 \leq \operatorname{Tr} \widetilde{W} \leq \pi / 2$ as required.

\footnotetext{
${ }^{8}$ For this purpose, we regard $L^{2}\left(\mathbb{R}^{+}, d \lambda\right) \otimes \mathbb{C}^{4}$ as $L^{2}(X, d \lambda \otimes d \mu)$ where $X$ is the locally compact space $\mathbb{R}^{+} \times \mathbb{Z}_{4}$ and $\mu$ is the counting measure on $\mathbb{Z}_{4}$. The measure $d \lambda \otimes d \mu$ is a Baire measure and the lemma may be applied.
} 


\section{Proof of Lemma 4.2}

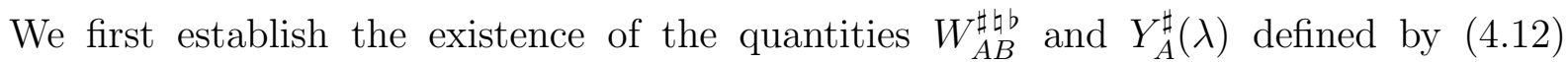
and (4.15). Using self-adjointness of $P^{\sharp}$, Cauchy-Schwarz and $\|Q\| \leq 1$ (following from property (I) of Sec. 2.3 for $Q \in \operatorname{Had}(\mathscr{H}, \Gamma)$ ) we obtain from (4.10) the inequality

$$
\left|\omega^{\sharp \text { म }}\left(f_{1} \otimes f_{2}^{+}\right)\right|^{2} \leq\left\|P^{\sharp} \Gamma\left[\begin{array}{c}
0 \\
f_{1}
\end{array}\right]\right\|^{2}\left\|P^{\mathrm{b}}\left[\begin{array}{c}
f_{2}^{+} \\
0
\end{array}\right]\right\|^{2}=\omega_{P}^{\sharp}\left(f_{1} \otimes f_{1}^{+}\right) \omega_{P}^{b}\left(f_{2} \otimes f_{2}^{+}\right)
$$

for any $f_{i} \in \mathscr{D}_{\mathrm{sp}}(i=1,2)$. This inequality underlies the following lemma, which will be proved at the end of this section.

Lemma 5.1 The wave-front set of $\omega^{\sharp \text { th }}$ satisfies

$$
\mathrm{WF}\left(\omega^{\sharp \text { घb }}\right) \subset \bigcup_{p, p^{\prime} \in M}\{p\} \times \mathcal{N}_{p}^{\sharp} \times\left\{p^{\prime}\right\} \times-\mathcal{N}_{p^{\prime}}^{b}
$$

as a subset of $T^{*}(M \times M)$.

This is by no means a sharp estimate of the wave-front set, but it will suffice for our purposes.

Defining $u_{A B}$ as in (4.2), $u_{A B} \omega^{\sharp \text { th }}$ is a scalar bi-distribution with wave-front set contained in the right-hand-side of Eq. (5.2). Now by Theorem 2.5.11' in [25], the pull-back $\gamma_{2}^{*} u_{A B} \omega^{\sharp \text { th }}$ exists provided the intersection of its wave-front set with the set of normals $N_{\gamma_{2}}$ of $\gamma_{2}$ is empty. One may show that

$$
N_{\gamma_{2}}=\left\{\left(\gamma(\tau), \xi ; \gamma\left(\tau^{\prime}\right), \xi^{\prime}\right) \in T^{*}(M \times M) \mid \xi_{a} u^{a}(\tau)=\xi_{b^{\prime}}^{\prime} u^{b^{\prime}}\left(\tau^{\prime}\right)=0\right\}
$$

(see Sec. 3 of [8] where a corresponding argument is given). This has trivial intersection with $\mathrm{WF}\left(u_{A B} \omega^{\sharp \text { bb }}\right)$, because no null covector can annihilate a timelike vector. Accordingly, $\gamma_{2}^{*} u_{A B} \omega^{\sharp \text { th }}$ exists in $\mathscr{D}^{\prime}\left(\mathbb{R}^{2}\right)$, and (again by Theorem 2.5.11' in [25]) its wave-front set obeys

$$
\mathrm{WF}\left(\gamma_{2}^{*} u_{A B} \omega^{\sharp \text { bb }}\right) \subset \mathbb{R} \times \mathbb{R}^{\sharp} \times \mathbb{R} \times-\mathbb{R}^{b} .
$$

Since $u_{A B}$ is compactly supported, we may take Fourier transforms and conclude that the $W_{A B}^{\sharp \sharp b}$ do indeed exist. Exactly the same argument, using the microlocal spectrum condition (2.50) in place of (5.2), shows that the pull-backs $\gamma_{2}^{*} u_{A B} \omega_{P}^{\sharp}$ exist with

$$
\mathrm{WF}\left(\gamma_{2}^{*} u_{A B} \omega_{P}^{\sharp}\right) \subset \mathbb{R} \times \mathbb{R}^{\sharp} \times \mathbb{R} \times-\mathbb{R}^{\sharp} .
$$

It remains to prove Lemmas 1.2 and 5.1 .

Proof of Lemma 4.9: An argument using regularising sequences in analogy with the proof of Theorem 2.2 in [8] shows that the inequality (5.1) is inherited by the pull-back and becomes

$$
\left|\gamma_{2}^{*} u_{A B} \omega^{\sharp \text { मb }}\left(f_{1} \otimes \overline{f_{2}}\right)\right|^{2} \leq \gamma_{2}^{*} u_{A A} \omega_{P}^{\sharp}\left(f_{1} \otimes \overline{f_{1}}\right) \gamma_{2}^{*} u_{B B} \omega_{P}^{b}\left(f_{2} \otimes \overline{f_{2}}\right) \quad \forall f_{1}, f_{2} \in C_{0}^{\infty}(\mathbb{R})
$$


(no sum on either $A$ or $B$ ). Substituting $f_{1}(t)=\mathrm{e}^{-i t \lambda}, f_{2}\left(t^{\prime}\right)=\mathrm{e}^{-i t^{\prime} \lambda^{\prime}}$, the required bounds (4.13) are obtained.

As $\gamma_{2}^{*} u_{A A} \omega_{P}$ is compactly supported, its set of singular directions (those directions in which its Fourier transform fails to decay rapidly) is given by

$$
\begin{aligned}
\Sigma\left(\gamma_{2}^{*} u_{A A} \omega_{P}^{*}\right) & =\left\{\left(\lambda, \lambda^{\prime}\right) \mid\left(\tau, \lambda ; \tau^{\prime}, \lambda^{\prime}\right) \in \mathrm{WF}\left(\gamma_{2}^{*} u_{A A} \omega_{P}^{\prime}\right) \text { for some }\left(\tau, \tau^{\prime}\right) \in \mathbb{R}^{2}\right\} \\
& =\mathbb{R}^{+} \times \mathbb{R}^{-}
\end{aligned}
$$

(see Proposition 8.1.3 in 24]). Thus $(-1,1)$ is not a singular direction for $\gamma_{2}^{*} u_{A A} \omega_{P}$ and we deduce that $Y_{\dot{A}}(\lambda)$ is rapidly decaying at $\lambda \rightarrow+\infty$ and of polynomially bounded growth as $\lambda \rightarrow-\infty$ by the Paley-Wiener-Schwartz theorem [24]. An analogous argument shows that $Y_{A}^{\Gamma}(\lambda)$ decays rapidly as $\lambda \rightarrow-\infty$ and is polynomially bounded as $\lambda \rightarrow+\infty$.

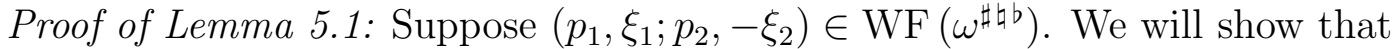

$$
\left(p_{1}, \xi_{1} ; p_{1},-\xi_{1}\right) \in \mathrm{WF}\left(\omega_{P}^{\sharp}\right) \quad \text { and } \quad\left(p_{2}, \xi_{2} ; p_{2},-\xi_{2}\right) \in \mathrm{WF}\left(\omega_{P}^{b}\right),
$$

from which the required result now follows by the microlocal spectrum condition (2.50). To prove (5.8) fix charts $\left(U_{i}, \kappa_{i}\right)$ with $p_{i} \in U_{i}(i=1,2)$, and define $k_{i}$ so that $\xi_{i}={ }^{t} \kappa_{i}^{\prime}\left(p_{i}\right) k_{i}$. Let $\chi_{i}$ be arbitrary smooth spinor fields compactly supported in $U_{i}$ with $\chi_{i}\left(p_{i}\right) \neq 0$. By definition of the wave-front set on manifolds, $\left(k_{1},-k_{2}\right)$ is a singular direction for $\chi_{12} \omega^{\sharp \text { }} \mathrm{b} \circ \kappa_{12}^{-1}$, where we use the notation

$$
\chi_{i j}=\chi_{i} \otimes \chi_{j}^{+} ; \quad \kappa_{i j}=\kappa_{i} \times \kappa_{j} .
$$

Thus, if $V_{j}$ are arbitrary conical neighbourhoods of $k_{j}$, there must exist $k_{j}^{\prime} \in V_{j}$ such that

$$
\left[\chi_{12} \omega^{\sharp \text { } b} \circ \kappa_{12}^{-1}\right]^{\wedge}\left(\alpha k_{1}^{\prime},-\alpha k_{2}^{\prime}\right)
$$

is not of rapid decay as $\alpha \rightarrow+\infty$. Applying (5.1) to

$$
f_{j}=\left(\frac{1}{\sqrt{-|\boldsymbol{g}|} \circ \kappa_{j}^{-1}} \chi_{j} \mathrm{e}^{i(.) \alpha k_{j}^{\prime}}\right) \circ \kappa_{j},
$$

and recalling (1.3), we obtain

$$
\left|\left[\chi_{12} \omega^{\sharp \text { 七b }} \circ \kappa_{12}^{-1}\right]^{\wedge}\left(\alpha k_{1}^{\prime},-\alpha k_{2}^{\prime}\right)\right| \leq\left[\chi_{11} \omega_{P}^{\sharp} \circ \kappa_{11}^{-1}\right]^{\wedge}\left(\alpha k_{1}^{\prime},-\alpha k_{1}^{\prime}\right)\left[\chi_{22} \omega_{P}^{b} \circ \kappa_{22}^{-1}\right]^{\wedge}\left(\alpha k_{2}^{\prime},-\alpha k_{2}^{\prime}\right)
$$

and since the left-hand side is not of rapid decrease as $\alpha \rightarrow \infty$ we conclude that the same must be true of both factors on the right-hand side. Hence (as the $V_{j}$ were arbitrary) $\left(k_{1},-k_{1}\right)$ is a singular direction for $\chi_{11} \omega_{P}^{\sharp} \circ \kappa_{11}^{-1}$ and $\left(k_{2},-k_{2}\right)$ is a singular direction for $\chi_{22} \omega_{P}^{b} \circ \kappa_{22}^{-1}$. Letting the support of each $\chi_{i}$ shrink to $\left\{p_{i}\right\}$, we have

$$
\left(\kappa\left(p_{1}\right), k_{1} ; \kappa\left(p_{1}\right),-k_{1}\right) \in \mathrm{WF}\left(\omega_{P}^{\sharp} \circ \kappa_{11}^{-1}\right)
$$

and

$$
\left(\kappa\left(p_{2}\right), k_{2} ; \kappa\left(p_{2}\right),-k_{2}\right) \in \mathrm{WF}\left(\omega_{P}^{b} \circ \kappa_{22}^{-1}\right),
$$

from which (5.8) follows immediately. 


\section{Proof of Proposition 4.4}

We now prove that the operators $\widetilde{J}_{\Lambda}$ on $L^{2}\left(\mathbb{R}^{+}, d \lambda\right) \otimes \mathbb{C}^{4}$ are bounded from below uniformly in $\Lambda$. To begin, we consider the related operator $K_{\Lambda}$ acting on $L^{2}\left(\mathbb{R}^{+}, d \lambda\right)$ by

$$
\left(K_{\Lambda} \varphi\right)(\lambda)=\int_{0}^{\infty} d \lambda \frac{\sigma_{\Lambda}(\lambda) \sigma_{\Lambda}\left(\lambda^{\prime}\right)}{8 \pi^{2}}\left(\lambda+\lambda^{\prime}\right) \widehat{f}\left(\lambda-\lambda^{\prime}\right) \varphi\left(\lambda^{\prime}\right) .
$$

If the spin-connection terms vanished, $\widetilde{J}_{\Lambda}$ would be equal to $K_{\Lambda} \otimes \mathbb{1}$. Our analysis of $K_{\Lambda}$ is based on the following identity.

Lemma 6.1 If $f=g^{2}$ for real-valued $g \in C_{0}^{\infty}(\mathbb{R})$, then

$$
\left(\lambda+\lambda^{\prime}\right) \widehat{f}\left(\lambda-\lambda^{\prime}\right)=\int_{-\infty}^{\infty} \frac{d \mu}{\pi} \mu \widehat{g}(\lambda-\mu) \overline{\widehat{g}\left(\lambda^{\prime}-\mu\right)} .
$$

Proof: Note first that the right-hand side (RHS) exists for each $\lambda, \lambda^{\prime} \in \mathbb{R}$. Changing variables to $\nu=\mu-\left(\lambda+\lambda^{\prime}\right) / 2$ and writing $\zeta=\left(\lambda-\lambda^{\prime}\right) / 2$

$$
\begin{aligned}
\text { RHS of (6.2) } & =\int_{-\infty}^{\infty} \frac{d \nu}{\pi}\left(\frac{\lambda+\lambda^{\prime}}{2}+\nu\right) \widehat{g}(\zeta-\nu) \widehat{g}(\zeta+\nu) \\
& =\left(\lambda+\lambda^{\prime}\right)(\widehat{g} \star \widehat{g})(2 \zeta) \\
& =\left(\lambda+\lambda^{\prime}\right) \widehat{f}\left(\lambda-\lambda^{\prime}\right)
\end{aligned}
$$

as required, where we have also used $\overline{\widehat{g}(u)}=\widehat{g}(-u)$, the fact that $\nu \widehat{g}(\zeta-\nu) \widehat{g}(\zeta+\nu)$ is odd, and a further change of variables. In addition, we have used $\star$ to denote the convolution $\left(h_{1} \star h_{2}\right)(\lambda)=\int d \lambda^{\prime} /(2 \pi) h_{1}\left(\lambda-\lambda^{\prime}\right) h_{2}\left(\lambda^{\prime}\right)$.

It follows from this identity that the kernel of $K_{\Lambda}$ may be rewritten in the form

$$
K_{\Lambda}\left(\lambda, \lambda^{\prime}\right)=\frac{\sigma_{\Lambda}(\lambda) \sigma_{\Lambda}\left(\lambda^{\prime}\right)}{8 \pi^{3}} \int_{-\infty}^{\infty} d \mu \mu \widehat{g}(\lambda-\mu) \overline{\widehat{g}\left(\lambda^{\prime}-\mu\right)} .
$$

We now define $K_{\Lambda}^{ \pm}$to have the kernels

$$
K_{\Lambda}^{+}\left(\lambda, \lambda^{\prime}\right)=\frac{\sigma_{\Lambda}(\lambda) \sigma_{\Lambda}\left(\lambda^{\prime}\right)}{8 \pi^{3}} \int_{-\infty}^{\infty} d \mu|\mu| \widehat{g}(\lambda-\mu) \overline{\widehat{g}\left(\lambda^{\prime}-\mu\right)}
$$

and

$$
K_{\Lambda}^{-}\left(\lambda, \lambda^{\prime}\right)=-\frac{2 \sigma_{\Lambda}(\lambda) \sigma_{\Lambda}\left(\lambda^{\prime}\right)}{8 \pi^{3}} \int_{-\infty}^{0} d \mu \mu \widehat{g}(\lambda-\mu) \overline{\widehat{g}\left(\lambda^{\prime}-\mu\right)}
$$

The integrals in these kernels are bounded on compact subsets of $\mathbb{R}^{+} \times \mathbb{R}^{+}$, so the cut-off functions $\sigma_{\Lambda}$ ensure that $K_{\Lambda}$ and $K_{\Lambda}^{ \pm}$are Hilbert-Schmidt. Clearly $K_{\Lambda}=K_{\Lambda}^{+}-K_{\Lambda}^{-}$; furthermore, the easily proven identity

$$
\left\langle\varphi \mid K_{\Lambda}^{-} \varphi\right\rangle=\int_{0}^{\infty} d \mu \frac{\mu}{4 \pi^{3}}\left|\int_{0}^{\infty} d \lambda^{\prime} \overline{\widehat{g}\left(\lambda^{\prime}+\mu\right)} \sigma_{\Lambda}\left(\lambda^{\prime}\right) \varphi\left(\lambda^{\prime}\right)\right|^{2}
$$


(valid, say, for $\varphi \in C_{0}^{\infty}\left(\mathbb{R}^{+}\right)$) shows that $K_{\Lambda}^{-}$is positive. A similar argument establishes positivity of $K_{\Lambda}^{+}$.

Our aim is now to find a bound on $K_{\Lambda}^{-}$which will allow a bound uniform in $\Lambda$ to be obtained. (The operator $K_{\Lambda}^{+}$is bounded for each $\Lambda$, e.g., by its Hilbert-Schmidt norm, but becomes unbounded in the limit $\Lambda \rightarrow \infty)$. Regarding the inner integral in (6.7) as an $L^{2}$-inner product and applying Cauchy-Schwarz, we obtain

$$
\left\langle\varphi \mid K_{\Lambda}^{-} \varphi\right\rangle \leq C_{\Lambda}\|\varphi\|^{2}
$$

where

$$
\begin{aligned}
C_{\Lambda} & =\frac{1}{4 \pi^{3}} \int_{\mathbb{R}^{+} \times \mathbb{R}^{+}} d \mu d \lambda^{\prime} \mu\left|\overline{\widehat{g}\left(\mu+\lambda^{\prime}\right)} \sigma_{\Lambda}\left(\lambda^{\prime}\right)\right|^{2} \\
& =\int_{0}^{\infty} d u|\widehat{g}(u)|^{2} F_{\Lambda}(u)
\end{aligned}
$$

and

$$
F_{\Lambda}(u)=\frac{1}{4 \pi^{3}} \int_{0}^{u} d \lambda^{\prime}\left(u-\lambda^{\prime}\right) \sigma_{\Lambda}\left(\lambda^{\prime}\right)^{2}
$$

is bounded and nonnegative. Let us observe that this step depends in an essential way on the fact that, for $\mu>0$, the argument of $\widehat{g}$ in (6.7) is bounded away from zero, together with the rapid decay property of $\widehat{g}$.

The above analysis entails that $-C_{\Lambda}$ is a lower bound for $K_{\Lambda}$, but this is certainly not the sharpest bound. In fact essentially the same argument applies if $K_{\Lambda}^{-}$is replaced by $\frac{1}{2} K_{\Lambda}^{-}$and $K_{\Lambda}^{+}$is adjusted to maintain $K_{\Lambda}=K_{\Lambda}^{+}-K_{\Lambda}^{-}$, with the conclusion that $-C_{\Lambda} / 2$ is also a lower bound for $K_{\Lambda}$. The convenience of the choices made above is that, as we now show, the operator $L_{\Lambda}=\widetilde{J}_{\Lambda}-K_{\Lambda} \otimes \mathbb{1}$ is form bounded relative to $K_{\Lambda}^{+}$with relative bound no greater than $\frac{1}{2}$. To this end, we first use the convolution theorem to write

$$
\left\langle\varphi \mid L_{\Lambda} \varphi\right\rangle=\frac{1}{2} \int_{-\infty}^{\infty} d \tau\left[\sigma_{\Lambda} \varphi\right]^{\vee}(\tau)^{\dagger} f(\tau) \theta(\tau)\left[\sigma_{\Lambda} \varphi\right]^{\vee}(\tau)
$$

for $\varphi \in C_{0}^{\infty}\left(\mathbb{R}^{+}\right) \otimes \mathbb{C}^{4}$, where $\dagger$ denotes the matrix hermitian conjugate. Setting $C^{\prime}=$ $\sup _{\tau \in \mathbb{R}}\|\theta(\tau)\|_{\mathbb{C}^{4}}$, we then estimate

$$
\begin{aligned}
\left|\left\langle\varphi \mid L_{\Lambda} \varphi\right\rangle\right| & \leq \frac{C^{\prime}}{2} \int_{-\infty}^{\infty} d \tau\left|\left(g\left[\sigma_{\Lambda} \varphi\right]^{\vee}\right)(\tau)\right|_{\mathbb{C}^{4}}^{2} \\
& \leq \frac{C^{\prime}}{2} \int_{-\infty}^{\infty} \frac{d \mu}{2 \pi}\left|\left(g\left[\sigma_{\Lambda} \varphi\right]^{\vee}\right)^{\wedge}(\mu)\right|_{\mathbb{C}^{4}}^{2} \\
& =\frac{C^{\prime}}{2} \int_{-\infty}^{\infty} \frac{d \mu}{2 \pi}\left|\int_{0}^{\infty} \frac{d \lambda^{\prime}}{2 \pi} \widehat{g}\left(\mu-\lambda^{\prime}\right) \sigma_{\Lambda}\left(\lambda^{\prime}\right) \varphi\left(\lambda^{\prime}\right)\right|_{\mathbb{C}^{4}}^{2}
\end{aligned}
$$

By comparison with the definition of $K_{\Lambda}^{+}$, this implies

$$
\left|\left\langle\varphi \mid L_{\Lambda} \varphi\right\rangle\right| \leq \frac{1}{2}\left\langle\varphi \mid\left(K_{\Lambda}^{+} \otimes \mathbb{1}\right) \varphi\right\rangle+\frac{C^{\prime}}{16 \pi^{3}} \int_{|\mu|<C^{\prime}} d \mu\left|\int_{0}^{\infty} d \lambda^{\prime} \widehat{g}\left(\mu-\lambda^{\prime}\right) \sigma_{\Lambda}\left(\lambda^{\prime}\right) \varphi\left(\lambda^{\prime}\right)\right|_{\mathbb{C}^{4}}^{2} .
$$


Using a similar argument to that used to obtain (6.8), the last term may be bounded by $C_{\Lambda}^{\prime \prime}\|\varphi\|^{2}$ where

$$
\begin{aligned}
C_{\Lambda}^{\prime \prime} & =\frac{C^{\prime}}{16 \pi^{3}} \int_{|\mu|<C^{\prime}} d \mu \int_{0}^{\infty} d \lambda^{\prime}\left|\overline{\widehat{g}\left(\mu+\lambda^{\prime}\right)} \sigma_{\Lambda}\left(\lambda^{\prime}\right)\right|^{2} \\
& =\int_{-C^{\prime}}^{\infty} d u|\widehat{g}(u)|^{2} G_{\Lambda}(u)
\end{aligned}
$$

and the bounded nonnegative function $G_{\Lambda}$ is given on $\left[-C^{\prime}, \infty\right)$ by

$$
G_{\Lambda}(u)=\frac{C^{\prime}}{16 \pi^{3}} \int_{\max \left\{u-C^{\prime}, 0\right\}}^{u+C^{\prime}} d \lambda^{\prime} \sigma_{\Lambda}\left(\lambda^{\prime}\right)^{2} .
$$

Thus $L_{\Lambda}$ is form bounded relative to $K_{\Lambda}^{+}$as claimed above. Since $\widetilde{J}_{\Lambda}=K_{\Lambda}^{+}-K_{\Lambda}^{-}+L_{\Lambda}$, we have

$$
\begin{aligned}
\left|\left\langle\varphi \mid\left(\widetilde{J}_{\Lambda}-K_{\Lambda}^{+} \otimes \mathbb{1}\right) \varphi\right\rangle\right| & \leq\left|\left\langle\varphi \mid L_{\Lambda} \varphi\right\rangle\right|+\left|\left\langle\varphi \mid K_{\Lambda}^{-} \varphi\right\rangle\right| \\
& \leq \frac{1}{2}\left\langle\varphi \mid\left(K_{\Lambda}^{+} \otimes \mathbb{1}\right) \varphi\right\rangle+\left(C_{\Lambda}+C_{\Lambda}^{\prime \prime}\right)\|\varphi\|^{2}
\end{aligned}
$$

and, as $K_{\Lambda}^{+}$is positive, it follows that $\widetilde{J}_{\Lambda}$ is bounded below by $-\left(C_{\Lambda}+C_{\Lambda}^{\prime \prime}\right)$.

To complete the proof of Proposition 4.4 we must show that $C_{\Lambda}$ and $C_{\Lambda}^{\prime \prime}$ may both be bounded above uniformly in $\Lambda$. This holds because $\sigma_{\Lambda}(\lambda)$ is pointwise dominated by $\sigma(\lambda)$ and thus $F_{\Lambda}$ and $G_{\Lambda}$ are pointwise dominated by the functions $F$ and $G$ obtained by replacing $\sigma_{\Lambda}$ by $\sigma$ in (6.10) and (6.15). Furthermore, $F$ and $G$ are of polynomially bounded growth so we obtain the bounds

$$
C_{\Lambda} \leq \int_{0}^{\infty} d u|\widehat{g}(u)|^{2} F(u)<\infty
$$

and

$$
C_{\Lambda}^{\prime \prime} \leq \int_{-C^{\prime}}^{\infty} d u|\widehat{g}(u)|^{2} G(u)<\infty
$$

for all $\Lambda$, where the rapid decay property of $\widehat{g}$ has been used.

\section{Majorana fields}

In this section we will indicate the changes required in Sec. 3 when treating Majorana fields. Suppose that $\gamma_{0}, \ldots, \gamma_{3}$ belong to a Majorana representation and that $\mathscr{H}$ is the completion of $\mathscr{D}_{\text {cosp }} /$ ker $S_{\triangleleft}$ with scalar product given by $\left(h, h^{\prime}\right)_{S}=\left(S_{\triangleleft} h, h^{\prime}\right)$, cf. Remark (iii) at the end of Sec. 2.2. The field operators are then given by

$$
\Psi(h)=B(q(h)) ; \quad \Psi^{+}(f)=\Psi\left(f^{+}\right)^{*}=\Psi\left(\Gamma f^{+}\right) \quad h \in \mathscr{D}_{\mathrm{cosp}}, f \in \mathscr{D}_{\mathrm{sp}}
$$

where $q: \mathscr{D}_{\text {cosp }} \rightarrow \mathscr{D}_{\text {cosp }} /$ ker $S_{\triangleleft}$ is the quotient map. Now, as in Sec. 3 one may choose, in a tubular neighbourhood of any timelike curve $\gamma$, induced frames $\left(e_{0}, \ldots, e_{3}\right)$ and $\left(E_{A}\right)$ 
so that $\left.e_{0}\right|_{\gamma}=u$, the tangent of $\gamma$ in proper time parametrization. However, it is now convenient to set $v_{A}=i E_{A}$, for then we have the two properties

$$
\delta^{A B} v_{A} \otimes v_{B}^{+}=\gamma_{0} \quad \text { and } \quad \Gamma v_{A}^{+}=v_{A}^{+}
$$

as we are working in a Majorana representation.

To quantize the point-split energy density (3.4), we may substitute from (7.1) and use the formula $\Gamma\left[\nabla \cdot\left(e_{0} v_{A} f\right)\right]^{+}=\nabla \cdot\left(f e_{0} v_{A}^{+}\right)$for $f \in \mathscr{D}(M)$ to obtain

$$
T(f \otimes g)=\delta^{A B} \frac{i}{2}\left(\Psi\left(\nabla \cdot\left[f e_{0} v_{A}^{+}\right]\right) \Psi\left(v_{B}^{+} g\right)-\Psi\left(f v_{A}^{+}\right) \Psi\left(\nabla \cdot\left[e_{0} v_{B}^{+} g\right]\right)\right)
$$

as the replacement for (3.6). The CAR's may be used to show that $\langle: T:\rangle_{\omega}(f \otimes g)$ is symmetric in $f, g$.

Using the formula $e_{0} \cdot \nabla v_{B}^{+}=\overline{\sigma_{0}{ }_{B}^{C}} v_{C}^{+}$, it follows that $\langle T\rangle_{\omega}$ may be expressed in the form

$$
\langle T\rangle_{\omega}=\mathcal{L}^{A B} v_{A}^{+} \otimes v_{B}^{+} \omega_{2},
$$

where $\omega_{2}\left(h, h^{\prime}\right)=\omega\left(\Psi(h) \Psi\left(h^{\prime}\right)\right)$ is the two-point function and

$$
\mathcal{L}^{A B}=\frac{1}{2}\left(\mathbb{1} \otimes i e_{0} \cdot \nabla-i e_{0} \cdot \nabla \otimes \mathbb{1}\right) \delta^{A B}+\frac{1}{2} \Theta^{A B},
$$

with

$$
\Theta^{A B}=i\left[\delta^{C B} \overline{\sigma_{0}{ }^{A} C} \otimes \mathbb{1}-\mathbb{1} \otimes \delta^{A C} \overline{\sigma_{0}{ }^{B} C}\right] .
$$

The components of $\sigma_{0}$ are real in a Majorana representation and it follows that $\theta^{A B}(\tau)=$ $\gamma_{2}^{*} \Theta^{A B}(\tau, \tau)$ is hermitian for each $\tau$. Moreover, $v_{A}^{+} \otimes v_{B}^{+} \omega_{2}$ is of matrix positive type as shown by the calculation

$$
\left(v_{A}^{+} \otimes v_{B}^{+} \omega_{2}\right)\left(\overline{f^{A}} \otimes f^{B}\right)=\omega_{2}\left(\Gamma\left[v_{A}^{+} f^{A}\right] \otimes v_{B}^{+} f^{B}\right)=\omega\left(\Psi\left(v_{A}^{+} f\right)^{*} \Psi\left(v_{B}^{+} f^{B}\right)\right) \geq 0
$$

in which we have used $\Gamma v_{A}^{+}=v_{A}^{+}$and $(7.1)$.

From this point onwards, one may proceed to define $\langle: \rho:\rangle_{\omega}$ as in Sec. 3, and the statement and proof of Thm. 4.1 carry over (apart from some obvious changes) upon observing that Hadamard states $\omega$ of the Majorana field obey

$$
\mathrm{WF}\left(\omega_{2}\right)=\left\{\left(p, \xi ; p^{\prime},-\xi^{\prime}\right) \in \dot{T}^{*}(M \times M) \mid(p, \xi) \sim\left(p^{\prime}, \xi^{\prime}\right) ; \quad \xi \in \mathcal{N}_{p}^{+}\right\}
$$

(cf. [38], note again that in this reference a different sign convention for the Fourier transform was chosen which results in the opposite form of the wave-front set there).

\section{Conclusion}

In this paper, we have established general QWEIs for the Dirac and Majorana fields in globally hyperbolic spacetimes. We conclude with various remarks. First, these QWEIs hold despite the fact that the 'classical' Dirac equation fails to obey the weak energy 
condition. This is encouraging evidence that QWEIs are a widespread feature of all quantum field theories and that they are the correct replacement for the classical energy conditions. It would be interesting to understand whether QWEIs can be obtained in a general axiomatic setting.

A second point was posed to us by Buchholz (private communication): given a weight in $\mathscr{W}$ the corresponding averaged energy density may be defined as a symmetric operator on a suitable dense domain (such as the domain of microlocal smoothness introduced in [2]) in some Hilbert space representation. The force of our result (and the corresponding result in [8]) is that such operators are semibounded, and therefore admit self-adjoint extensions (in particular, the Friedrichs extension). Can one give any interpretation to the evolution generated by this operator? The answer is not clear, but we speculate that there could be a connection with the dynamics discussed by Keyl [27] in his recent study of quantum fields on timelike curves. The connection is somewhat tentative (in particular, the role of the weight must be understood), and would only be expected to hold under restricted conditions such as for static trajectories in static spacetimes. Nonetheless, it remains an intriguing possibility.

Finally, although our approach does lead to explicit lower bounds on the various contributions to the averaged energy density, these are not expected to be optimal. We hope to return to this question elsewhere.

Acknowledgments: We thank the organisers of the meeting on Microlocal Analysis and Quantum Field Theory at the Mathematisches Forschungsinstitut Oberwolfach, where this work was commenced. CJF thanks Simon Eveson, Alfredo Calvo Pereira and Stefan Hollands for useful discussions, and is grateful to the Institut für Theoretische Physik in Göttingen for hospitality in the later stages of the work. We also thank Detlev Buchholz for raising the issue discussed above. The work of CJF was assisted by a grant from the Nuffield Foundation.

\section{References}

[1] Araki, H., "On quasifree states of CAR and Bogoliubov transformations", Publ. RIMS 6, $385(1970 / 71)$

[2] Brunetti, R., Fredenhagen, K., "Microlocal analysis and interacting quantum field theories: renormalization on physical backgrounds", Commun. Math. Phys. 208, 623 (2000)

[3] Davies, P.C.W., Fulling, S.A., "Radiation from moving mirrors and from black holes", Proc. Roy. Soc. A356, 237 (1977)

[4] Deser, S., "Improvement versus stability in gravity-scalar coupling", Phys. Lett. 134B, 419 (1984)

[5] Dimock, J., "Dirac quantum fields on a manifold", Trans. Am. Math. Soc. 269, 133 (1982)

[6] Epstein, H., Glaser, V., Jaffe, A., "Nonpositivity of the energy density in quantized field theories", Nuovo Cimento 36, 1016 (1965)

[7] Fewster, C.J., Eveson, S.P., "Bounds on negative energy densities in flat spacetime", Phys. Rev. D 58, 084010 (1998) 
[8] Fewster, C.J., "A general worldline quantum inequality", Class. Quantum Grav. 17, 1897 (2000)

[9] Fewster, C.J., Teo, E., "Bounds on negative energy densities in static spacetimes", Phys. Rev. D 59, 104016 (1999)

[10] Fewster, C.J., Teo, E., "Quantum inequalities and quantum interest as eigenvalue problems", Phys. Rev. D 61, 084012 (2000)

[11] Flanagan, É.É., "Quantum inequalities in two-dimensional Minkowski spacetime", Phys. Rev. D 56, 4922 (1997)

[12] Flanagan, É.É., Wald, R.M., "Does backreaction enforce the averaged null energy condition in semiclassical gravity?", Phys. Rev. D 54, 6233 (1996)

[13] Folacci, A., "Averaged-null-energy condition for electromagnetism in Minkowski spacetime", Phys. Rev. D 46, 2726 (1992)

[14] Ford, L.H., "Quantum coherence effects and the second law of thermodyamics", Proc. Roy. Soc. Lond. A364, 227 (1978)

[15] Ford, L.H., Roman, T.A., "Averaged energy conditions and quantum inequalities", Phys. Rev. D 51, 4277 (1995)

[16] Ford, L.H., Roman, T.A., "Restrictions on negative energy density in flat spacetime", Phys. Rev. D 55, 2082 (1997)

[17] Ford, L.H., Roman, T.A., "Classical Scalar Fields and the Generalized Second Law", arXiv:gr-qc/0009076

[18] Ford, L.H., Roman, T.A., "Quantum field theory constrains traversable wormhole geometries", Phys. Rev. D 53, 5496 (1996)

[19] Fulling, S.A., Narcowich, F.J., Wald, R.M., "Singularity structure of the two-point function in quantum field theory in curved spacetime, II", Ann. Phys. (N.Y.) 136, 243 (1981)

[20] Glaeser, G., "Racine carrée d'une fonction différentiable", Ann. Inst. Fourier, Grenoble 13, 203 (1963)

[21] Hawking, S.W., Ellis, G.F.R., The large scale structure of space-time, Cambridge University Press, Cambridge, 1973

[22] Helfer, A.D., "The Hamiltonians of Linear Quantum Fields: II. Classically Positive Hamiltonians", arXiv:hep-th/9908012

[23] Hollands, S., "The Hadamard condition for Dirac fields and adiabatic states on RobertsonWalker spacetimes", Commun. Math. Phys. 216, 635 (2001)

[24] Hörmander, L., The analysis of linear partial differential operators I, Springer Verlag, Berlin, 1983

[25] Hörmander, L., "Fourier integral operators. I", Acta Math. 127, 79 (1971) 
[26] Klinkhammer, G., "Averaged energy conditions for free scalar fields in flat space-time", Phys. Rev. D 43, 2542 (1991)

[27] Keyl, M., "Quantum fields on timelike curves", arXiv:math-ph/0012024

[28] Köhler, M., The stress energy tensor of a locally supersymmetric quantum field on a curved spacetime, Dissertation, Hamburg University, 1995. Preprint DESY-95-080, arXiv:grqc/9505014

[29] Kratzert, K., "Singularity structure of the two-point function of the free Dirac field on a globally hyperbolic spacetime", Annalen Phys. 9, 475 (2000)

[30] Najmi, A.-H., Ottewill, A.C., "Quantum states and the Hadamard form II, Energy minimization for spin 1/2 fields", Phys. Rev. D 30, 2573 (1984)

[31] Pfenning, M.J., Quantum inequality restrictions on negative energy densities in curved spacetimes, Ph.D. thesis, Tufts University, 1998. Preprint arXiv:gr-qc/9805037

[32] Pfenning, M.J., "Quantum inequalities for the electromagnetic field", in preparation (2001)

[33] Pfenning, M.J., Ford, L.H., "The unphysical nature of "warp drive"", Class. Quantum Grav. 14, 1743 (1997)

[34] Pfenning, M.J., Ford, L.H., "Scalar field quantum inequalities in static spacetimes", Phys. Rev. D 57, 3489 (1998)

[35] Radzikowski, M.J., "Micro-local approach to the Hadamard condition in quantum field theory in curved spacetime", Commun. Math. Phys. 179, 529 (1996)

[36] Reed, M., Simon, B., Methods of modern mathematical physics, vol III, Academic Press, San Diego, 1979

[37] Sahlmann, H., Verch, R., "Passivity and microlocal spectrum condition", Commun. Math. Phys. 214, 705 (2000)

[38] Sahlmann, H., Verch, R., "Microlocal spectrum condition and Hadamard form for vectorvalued quantum fields in curved spacetime", Rev. Math. Phys., to appear, arXiv:math$\mathrm{ph} / 0008029$

[39] Schoen, R., Yau, S.-T., "Proof of the positive mass theorem. II.", Commun. Math. Phys. 79, 231 (1981)

[40] Taylor, M.E., Pseudodifferential operators, Princeton University Press, Princeton, 1981

[41] Tipler, F.J., "Energy conditions and spacetime singularities", Phys. Rev. D 17, 2521 (1978)

[42] Verch, R., "The averaged null energy condition for general quantum field theories in two dimensions", J. Math. Phys. 41, 206 (2000)

[43] Visser, M., Barcelo, C., "Energy conditions and their cosmological implications", arXiv:grqc/0001099 
[44] Vollick, D.N., "Negative energy density states for the Dirac field in flat space-time", Phys. Rev. D 57, 3484 (1998)

[45] Vollick, D.N., "Quantum inequalities in curved two dimensional spacetime", Phys. Rev. D 61, $084022(2000)$

[46] Wald, R.M., Yurtsever, U., "General proof of the averaged null energy condition for a massless scalar field in two-dimensional curved spacetime", Phys. Rev. D 44, 403 (1991)

[47] Weinless, M., "Existence and uniqueness of the vacuum for linear quantized fields", J. Funct. Anal. 4, 350 (1969)

[48] Witten, E., "A new proof of the positive energy theorem", Commun. Math. Phys. 80, 381 (1981)

[49] Yurtsever, U., "Averaged null energy condition and difference inequalities in quantum field theory", Phys. Rev. D 51, 5797 (1995)

[50] Yurtsever, U., "Remarks on the averaged null energy condition in quantum field theory", Phys. Rev. D 52, R564 (1995) 